\title{
A Computational Model for the Influence of Corollary Discharge and Proprioception on the Perisaccadic Mislocalization of Briefly Presented Stimuli in Complete Darkness
}

\author{
Arnold Ziesche ${ }^{1,2,3}$ and Fred H. Hamker ${ }^{1,2}$ \\ ${ }^{1}$ Chemnitz University of Technology, 09107 Chemnitz, Germany, ${ }^{2}$ Otto Creutzfeldt Center for Cognitive and Behavioral Neuroscience, 48149 Münster, \\ Germany, and ${ }^{3}$ Max Planck Institute for Human Cognitive and Brain Sciences, 04103 Leipzig, Germany
}

Spatial perception, the localization of stimuli in space, can rely on visual reference stimuli or on egocentric factors such as a stimulus position relative to eye gaze. In total darkness, only an egocentric reference frame provides sufficient information. When stimuli are briefly flashed around saccades, the localization error reveals potential mechanisms of updating such reference frames as described in several theories and computational models. Recent novel experimental evidence, however, showed that the maximum amount of mislocalization does not scale linearly with saccade amplitude but rather stays below $13^{\circ}$ even for long saccades, which is different from predicted by present models. We proposea new model of perisaccadic mislocalization in complete darkness to account for this observation. According to this model, mislocalization arises not on the motor side by comparing a retinal position signal with an extraretinal eye position related signal but by updating stimulus position in visual areas through a combination of proprioceptive eye position and corollary discharge. Simulations with realistic input signals and temporal dynamics show that both signals together are used for spatial updating and in turn bring about perisaccadic mislocalization.

\section{Introduction}

Our eyes move to sample the environment with high resolution at the center of gaze. This advantage comes with the cost of a fragmented visual input stream leading to the problem of maintaining visual stability (Wurtz, 2008). While visual input changes with each eye movement, our experience of the visual world is rather stable and not centered on the individual views. One line of research to study visual stability has focused on the localization of perisaccadically flashed stimuli in total darkness. It has been observed that, even before saccade onset, briefly flashed stimuli are mislocalized in saccade direction. The magnitude of misperception depends mainly on the interval between saccade onset and stimulus flash, regardless of stimulus position (Matin et al., 1970; Honda, 1989, 1991; Dassonville et al., 1992; Schlag and SchlagRey, 2002). Different models have been developed using the concept of a continuous eye position signal. Dassonville et al. (1992) suggested that, since misperceptions already occur presaccadi-

\footnotetext{
Received July 5, 2011; revised Aug. 20, 2011; accepted Sept. 12, 2011.

Author contributions: A.Z. and F.H.H. designed research; A.Z. and F.H.H. performed research; A.Z. and F.H.H. analyzed data; A.Z. and F.H.H. wrote the paper.

This work was supported by the Federal Ministry of Education and Research Grant "Visuospatial Cognition" (Bundesministerium für Bildung und Forschung Grant 01GW0653). We thank Marc Zirnsak and Adam Morris for helpful comments on a previous version of this manuscript. Moreover, we are grateful to John Van Opstal and Michael E. Goldberg for providing us data to replot their main experimental observations.

Correspondence should be addressed to Fred H. Hamker, Künstliche Intelligenz, Informatik, Technische Universität Chemnitz, Strasse der Nationen 62, 09107 Chemnitz, Germany. E-mail: fred.hamker@informatik. tu-chemnitz.de.

DOI:10.1523/JNEUROSCI.3407-11.2011

Copyright $\odot 2011$ the authors $\quad 0270-6474 / 11 / 3117392-14 \$ 15.00 / 0$
}

cally, the eye position information is sluggish and anticipates the saccade. Pola (2004) proposed a more elaborate model accounting for delay and visual persistence and concluded that the extraretinal signal may only change after saccade onset. Similarly, imposing the additional constraint of no mislocalization for continuously visible stimuli, Teichert et al. (2010) proposed that reafferent position information is sufficient to explain perisaccadic shift.

However, the mapping of these rather abstract models onto brain structures and mechanisms is still unidentified (Hamker et al., 2011). Moreover, an electrophysiological correlate of a psychophysical, continuous eye position signal is still lacking, although eye position-dependent responses are known to exist in different brain areas, as the parietal cortex (Andersen et al., 1990; Galletti et al., 1993; Bremmer et al., 1997). A correlate of eye position has been identified in monkey somatosensory cortex (Wang et al., 2007). However, this signal neither anticipates the eye movement nor does it shift continuously but rather jumps from the previous to the new fixation. A corollary discharge, here a copy of a motor command from the superior colliculus, can provide anticipatory information about upcoming eye movements (Sommer and Wurtz, 2004). However, since it encodes the saccade displacement, it does not hold as a continuous eye position signal.

In addition to the anatomical and physiological uncertainties, a problem of present models of perisaccadic localization in total darkness was recently discovered by Van Wetter and Van Opstal (2008). They found that the maximum amount of mislocalization saturates around $12^{\circ}$ even for long saccades up to $35^{\circ}$, whereas current models predict that mislocalization scales linearly with saccade amplitude (Van Wetter and Van Opstal, 2008). 

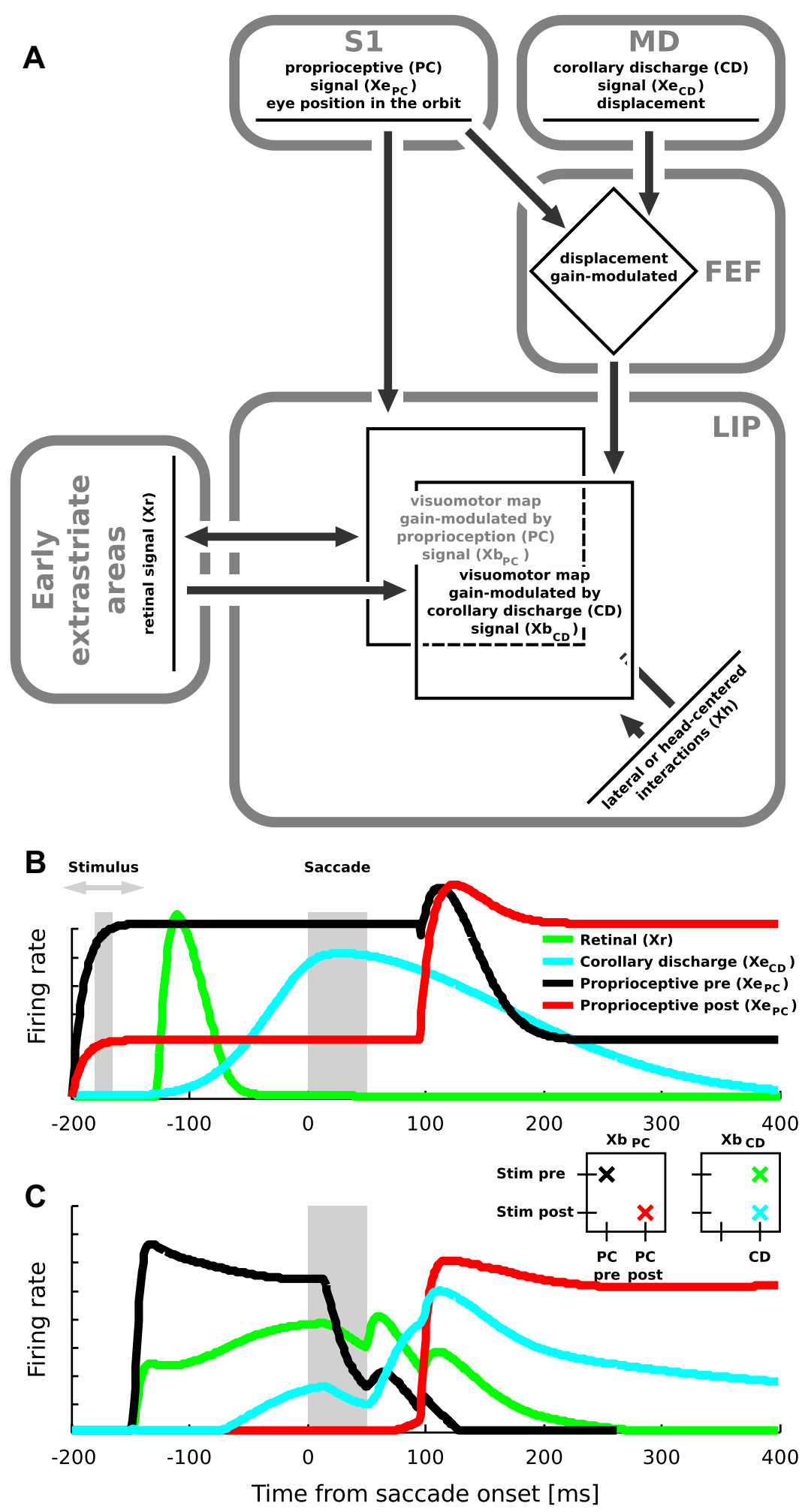

Figure 1. A, Our model of perisaccadic shift is composed of two cell types (in $L I P),{X b_{P C}}_{P C}$ and $X_{C D}$, which receive retinal input from early extrastriate areas as well as eye position information presumably from S1 and FEF. The input layer (Xr) represents stimulus position retinotopically in a single dimension, modeled by Gaussian receptive fields whose width depend linearly on the eccentricity of the stimulus (Hamker et al., 2008). The stimulus signal is gain modulated in $\mathrm{Xb}_{\mathrm{PC}}$ by the $\mathrm{PC}$ eye position signal $\left(\mathrm{Xe}_{\mathrm{PC}}\right)$. Similarly, another set of cells are gain modulated in $\mathrm{Xb}_{\mathrm{CD}}$ by the corollary discharge $\left(\mathrm{Xe}_{\mathrm{CD}}\right)$. To allow lateral (or alternatively feedback) interactions between the maps $\mathrm{Xb}_{\mathrm{PC}}$ and $\mathrm{Xb}_{\mathrm{CD}}$, both must encode space in the same coordinate system. Thus, the corollary discharge must implicitly encode eye position information, as motivated by an observation of Cassanello and Ferrera (2007). For simplicity, we use the same eye position signal $\left(X_{\mathrm{PC}}\right)$ to modulate corollary discharge from MD (and SC) with eye position. $\boldsymbol{B}$, Time courses of the input signals for a $15 \mathrm{~ms}$ stimulus flash and a $12^{\circ}$ saccade. Activities are shown for a Xr neuron tuned to the stimulus position (green), a $\mathrm{Xe}_{C D}$ neuron tuned to the saccade displacement (blue), and two $\mathrm{Xe}_{\mathrm{PC}}$ neurons tuned to presaccadic
Based on electrophysiological observations of corollary discharge (for review, see Sommer and Wurtz, 2008) and eye position in primary somatosensory cortex, we developed a novel model of perisaccadic perception in total darkness using a combination of eye position and corollary discharge. It explains the error in localization, the saturation of its magnitude, and a reduction of its magnitude with increased stimulus duration from the temporal dynamics in the model as the eyerelated signals change around saccade.

\section{Materials and Methods}

Our model for perisaccadic localization (Fig. 1A) is assumed to be located in the lateral intraparietal area (LIP). LIP is known to have neurons with retinocentric receptive fields that are modulated by eye position (Andersen et al., 1990; Bremmer et al., 1997) and saccade plans (Colby et al., 1996; Kusunoki and Goldberg, 2003), which makes it a likely area for the computations described in our proposed model. In this model, we simulate two different kinds of neurons in LIP. Both neuron types have retinocentric receptive fields. However, one type of neurons is gain modulated by a

$\leftarrow$

(black) and postsaccadic (red) eye positions. In Xr, neural latency (50 ms) and persistence are accounted for. During fixation, $\mathrm{Xe}_{\mathrm{PC}}$ encodes the eye position with a Gaussian of fixed width. Thirty-two milliseconds after saccade, the activity at the previous fixation starts to decay (after a brief rise) and increases at the same time at the postsaccadic eye position. Alignment to saccade offset is justified by Wang et al. (2007); however, alignment to saccade onset would also be possible. The $C D$ is modeled by a Gaussian with a fixed spatial width, consistent with a previous modeling study (Hamker et al., 2008) and peaks $10 \mathrm{~ms}$ after saccade onset (Sommer and Wurtz, 2004). Its time course is modeled by a Gaussian as well using a different length for increase and decrease as suggested by cell recordings (Sommer and Wurtz, 2004). C, Activity of selected RBF neurons for a constant stimulus and a $12^{\circ} \mathrm{sac}-$ cade. Black, $\mathrm{Xb}_{\mathrm{PC}}$ neuron with its receptive field (RF) center at the presaccadic stimulus position and sensitive to the initial eye position. Red, $\mathrm{Xb}_{\mathrm{PC}}$ neuron with its RF located at the postsaccadic stimulus position and sensitive to the postsaccadic $P C$. $G$ reen, $\mathrm{Xb}_{C D}$ neuron with its $\mathrm{RF}$ located at the presaccadic stimulus position and sensitive to the $C D$ for an eye displacement of $12^{\circ}$. Blue, $\mathrm{Xb}_{C D}$ neuron with its $\mathrm{RF}$ at the postsaccadic stimulus position and sensitive to the same $C D$. This $\mathrm{Xb}_{\mathrm{CD}}$ shows predictive remapping since it responds to the stimulus at the postsaccadic position already before saccade. Gray area, Duration of the saccade. The insets illustrate from which neurons the simulated activities are. The vertical axes show retinotopic stimulus position; the horizontal axes show the craniotopic proprioceptive signal and the corollary discharge signal. Left inset, Recorded cells in the $\mathrm{Xb}_{\mathrm{PC}}$ map. One neuron responds to stimuli in the presaccadic retinotopic position and is modulated by the presaccadic proprioceptive signal (black cross); the other responds to stimuli at the postsaccadic retinotopic position and is modulated by the postsaccadic proprioceptive signal (red cross). Right inset, Recorded cells in $\mathrm{Xb}_{\mathrm{CD}}$. One responds to the stimulus at the presaccadic retinotopic position (green cross), and the other, to the stimulus at the postsaccadic position (blue cross). Both are modulated by the $C D$ signal. 
proprioceptive (PC) eye position signal, which encodes the eye position in the orbit but does not update simultaneously with the eyes during saccades. The eye position signal of these neurons updates after saccade, similar to the updating of gain fields in LIP (Xu et al., 2010). Such a proprioceptive eye position signal could arise in somatosensory cortex (Wang et al., 2007), or alternatively, in the central thalamus (Tanaka, 2007). The other neurons in the model LIP are gain modulated by a corollary discharge (CD), which is assumed to originate in the superior colliculus (SC) (Sommer and Wurtz, 2004, 2008). It encodes eye displacement (i.e., retinotopic position of the saccade target) and is active around saccade onset. The $\mathrm{CD}$ is probably routed via the frontal eye field (FEF) where it is subject to an eye position gain field (Cassanello and Ferrera, 2007), which we assume to be also driven by the proprioceptive signal. This implicit eye position information in the $\mathrm{CD}$ signal is required to allow a combination of eye in the orbit with eye displacement information by lateral interactions as will be explained in more detail later. The activity of all simulated LIP neurons is decoded to determine the response of the model (i.e., the perceived spatial position) by a multiple-option diffusion model with decision neurons to obtain a spatial percept (Usher and McClelland, 2001; Hamker, 2007). As justified by empirical studies (Kiani et al., 2008; Stanford et al., 2010), this approach has the important advantage of accumulating varying evidence over time compared with time averages or snapshots. However, we do not necessarily assume that such decoding takes place in parietal cortex. Due to the anticipatory CD, some of the simulated LIP cells are modulated already presaccadically consistent with previous observations (Duhamel et al., 1992; Colby et al., 1996; Kusunoki and Goldberg, 2003).

Neurons are modeled by differential equations using rate coding, which allows for direct comparisons of model neural activity with neural recordings (for details, see Fig. $1 B, C$ ).

Such a modeling framework has previously been successfully applied in modeling attention (Hamker, 2005; Zirnsak et al., 2011). Eye movements are simulated by the saccade generator from Van Wetter and Van Opstal (2008).

Model details and parameters. We have implemented two variants of our model (Fig. 2). The main model (which we call non-head-centered model) does not have any explicit head-centered representation of stimulus position. For comparison, the second model variant (the headcentered model) contains an explicit head-centered neuron layer. Depending on the variant, our model consists of five or six maps.

A proprioceptive eye position signal is encoded in $\mathrm{Xe}_{\mathrm{PC}}$ (the " $\mathrm{e}$ " in "Xe" stands for eye position). In addition to proprioceptive eye position, a similar map $\mathrm{Xe}_{\mathrm{CD}}$ encodes the $\mathrm{CD}$ signal as it comes from the $\mathrm{SC}$ via the FEF. The CD signal is encoded retinotopically in SC and FEF but is subject to an eye position gain field in FEF (Cassanello and Ferrera, 2007), which we simulate in a layer $\mathrm{Xe}_{\mathrm{FEF}}$ and which is driven by the eye position signal in $\mathrm{Xe}_{\mathrm{PC}}$.

A retinotopic map Xr encodes the visual stimulus position information in retinal coordinates (the " $r$ " in "Xr" stands for retinal). The map $\mathrm{Xr}$ is assumed to model extrastriate visual areas, such as MT or V4. It projects to two two-dimensional maps $\mathrm{Xb}_{\mathrm{PC}}$ and $\mathrm{Xb}_{\mathrm{CD}}$ (the " $\mathrm{b}$ " in "Xb" stands for basis function). In $\mathrm{Xb}_{\mathrm{PC}}$, it interacts with the $\mathrm{PC}$ eye position signal from $\mathrm{Xe}_{\mathrm{PC}}$ to implement a radial basis function (RBF) (Denéve et al., 2001), a joint representation of stimulus position and eye position, which is known to exist in several parietal areas including LIP (Andersen et al., 1990; Bremmer et al.,
1997). Similarly, the stimulus is combined with the corollary discharge $\mathrm{Xe}_{\mathrm{FEF}}$, but slightly different, since corollary discharge is only phasically active around the eye movement. If one would record from a single cell of this combined layer $\mathrm{Xb}_{\mathrm{CD}}$, it would appear to the experimenter as a presaccadic (attentive) change in gain. As a result, $\mathrm{Xb}_{\mathrm{CD}}$ encodes a joint representation of stimulus position and eye displacement. Since both maps, $\mathrm{Xb}_{\mathrm{PC}}$ and $\mathrm{Xb}$ encode stimulus position in the same reference system, but using different eye-related signals, they can interact with each other either by lateral connections between these maps (non-head-centered model) or via an explicit head-centered representation of the stimulus in a map Xh (the " $\mathrm{h}$ " in "Xh" stands for head-centered) using feedback projections (head-centered model). These lateral or feedback connections are also relevant for anticipatory responses to stimuli placed in the future receptive field, known as remapping (Duhamel et al., 1992)

We simulate all one-dimensional layers with $n=40$ neurons and all two-dimensional layers with $n$ neurons along each dimension resulting in a total of $n^{2}$ neurons for each of these layers. We simulate a visual field of $v=160^{\circ}$, ranging from $-\frac{v}{2}$ to $\frac{v}{2}$. For all neurons, negative activity values are set to zero.

Retinotopic map Xr. We use Gaussian functions to model the receptive fields. Let $c_{i}^{\mathrm{Xr}}$ be the position of the receptive field center (i.e., the point in visual space that maximally activates the cell $i$ in $\mathrm{Xr}$ ). The width of the receptive field $\sigma^{\mathrm{Xr}}=\sigma^{\mathrm{Xr}}(\varepsilon)=b_{\mathrm{Xr}}+m_{\mathrm{Xr}} \varepsilon$ is a function of the eccentricity $\varepsilon$ ( $\varepsilon$ in degrees, $b_{\mathrm{Xr}}=6.35^{\circ}, m_{\mathrm{Xr}}=0.0875$ ). Let $p_{s}$ be the position of the 
stimulus in the visual field. For simplicity, we ignore the stimulus width. The sensory (bottom-up) input $r_{i}^{\mathrm{X} \text {,in }}$ of a given cell $i$ in $\mathrm{Xr}$ is then defined by the following:

$$
r_{i}^{\mathrm{Xr}, \text { in }}=S^{\mathrm{Xr}} c_{r} \exp \frac{-\left\|p_{s}-c_{i}^{\mathrm{Xr}}\right\|^{2}}{2\left(\sigma^{\mathrm{Xr}}(\varepsilon)\right)^{2}} .
$$

Note that $\left\|p_{s}-c_{i}^{\mathrm{X}}\right\|$ denotes the distance between the stimulus position and the receptive field center. $c_{r}=0.3$ is a contrast value (stimulus strength).

The response of the simulated neurons follows the stimulus onset with a latency $t_{\mathrm{Xr}}=50 \mathrm{~ms}$. The response strength decays over time while the stimulus is shown. This short-term synaptic depression $S^{\mathrm{Xr}}$ is simulated as in the study by Hamker (2005) as follows:

$$
\begin{gathered}
\tau_{S}^{\mathrm{Xr}}=\frac{d}{d t} s=1-s \\
S^{\mathrm{Xr}}=1-d_{S}^{\mathrm{Xr}} s,
\end{gathered}
$$

with time constant $\tau_{S}^{\mathrm{Xr}}=40$ and depression strength $d_{S}^{\mathrm{Xr}}=0.8$. After the stimulus is turned off, the input to Xr decays linearly for $d_{\mathrm{Xr}}=40 \mathrm{~ms}$ with a slope of $s_{\mathrm{Xr}}=0.025$ to account for a sufficiently long and realistic stimulus persistence.

The activity of a given $\mathrm{Xr}$ cell $i$ is given by the following ordinary differential equation (ODE):

$$
\tau \frac{d}{d t} r_{i}^{\mathrm{Xr}}=\underbrace{r_{i}^{\mathrm{Xr}, \text { in }}}_{\text {input }}(1+\underbrace{\left[A^{\mathrm{Xr}}-r_{i}^{\mathrm{Xr}}\right]}_{\text {saturation }}+\underbrace{\sum_{l, m} w_{i l m}^{\mathrm{Xb}}, \mathrm{Xr} r_{l m}^{\mathrm{Xb}}}_{\text {FB from } \mathrm{Xb}_{\mathrm{PC}}})-r_{i}^{\mathrm{Xr}} .
$$

Equation 4 is a function of the input $r_{i}^{\mathrm{Xr} \text {,in }}$, the feedback from $\mathrm{Xb}_{\mathrm{PC}}$ and a saturation factor $\left[A^{\mathrm{Xr}}-r_{i}^{\mathrm{Xr}}\right]^{+}$(Hamker, 2005). For the weights of the feedback connection from $\mathrm{Xb}_{\mathrm{PC}} w_{i l m}^{\mathrm{XbP}, \mathrm{Xr}}$, see below (see Basis function map $\left.\mathrm{Xb}_{\mathrm{PC}}\right) .\left[A^{\mathrm{Xr}}-r_{i}^{\mathrm{Xr}}\right]^{+}$with $A^{\mathrm{Xr}}=0.5$ implements a saturation of the gain for high-contrast stimuli, since the expression is zero for negative arguments (Hamker, 2005).

$P C$ eye position map $X e_{P C}$. We use a Gaussian input signal to model the population response of neurons in the proprioceptive eye position map $\mathrm{Xe}_{\mathrm{PC}}$ for a given eye position. The input to a cell $i$ in $\mathrm{Xe}_{\mathrm{PC}}$ is as follows:

$$
r_{i}^{\mathrm{Xe}, \text {,in }}=c_{\mathrm{PC}} \exp \frac{-\left\|c_{i}^{\mathrm{XepC}}-c^{\mathrm{PC}}\right\|^{2}}{2\left(\sigma^{\mathrm{PC}}\right)^{2}} .
$$

$c_{i}^{\mathrm{XePC}}$ is the position of the receptive field center of cell $i$ and $c^{\mathrm{PC}}$ is the eye position to be encoded. Note, that $c^{\mathrm{PC}}$ does not follow the eyes during saccades, but switches from the presaccadic to the postsaccadic position. $c_{\mathrm{PC}}=0.3$ is the strength of the proprioceptive signal, and $\sigma^{\mathrm{PC}}=8^{\circ}$ is its width.

The firing rate of $\mathrm{Xe}_{\mathrm{PC}}$ neurons is controlled by the following ODE:

$$
\tau \frac{d}{d t} r_{i}^{\mathrm{XePC}}=r_{i}^{\mathrm{XeP,in}}-r_{i}^{\mathrm{XePC}} .
$$

The proprioceptive signal at the postsaccadic eye position is turned on $t_{\mathrm{PC} \text {,on }}=32 \mathrm{~ms}$ relative to saccade offset, and the proprioceptive signal at the presaccadic eye position is turned off at the same time, also $t_{\mathrm{PC}, \text { off }}=$ $32 \mathrm{~ms}$ relative to saccade offset similar to the study by Wang et al. (2007). After the proprioceptive signal is turned off, it decays with a Gaussian decay factor $S_{\mathrm{PC} \text {,off }}$, which is introduced by replacing the input signal $r_{i}^{\mathrm{Xep}, \text { in }}$ in Equation 6 by the following:

$$
r_{i}^{\mathrm{Xe}, \text { in,offset }}=S_{\mathrm{PC}, \text { off }} r_{i}^{\mathrm{XePC}, \text { in }}
$$

with

$$
S_{\mathrm{PC}, \text { off }}=\exp \frac{-\|\Delta t\|^{2}}{2\left(\sigma_{\mathrm{PC}, \text { off }}\right)^{2}},
$$

where $\Delta t$ is the time relative to signal offset and $\sigma_{\mathrm{PC}, \text { off }}=35$.

Note that the saccade offset is calculated by a threshold of $22 \%$ on the eye velocity (i.e., when it is almost completely at rest). By using a more conservative value here, the timings of $t_{\mathrm{PC} \text {,on }}$ and $t_{\mathrm{PC} \text {,off }}$ for signal change would become even larger.

Basis function map $\mathrm{X} b_{P C}$. The proprioceptive eye position map $\mathrm{Xe}_{\mathrm{PC}}$ and the retinotopic map $\mathrm{Xr}$ encoding the stimulus are connected to the basis function map $\mathrm{Xb}_{\mathrm{PC}}$. The corresponding matrix of connection weights between a cell $i$ in $\mathrm{Xr}$ and a cell $(l, m)$ in $\mathrm{Xb}_{\mathrm{PC}}$ is as follows:

$$
\begin{aligned}
& w_{i l m}^{\mathrm{Xr}, \mathrm{XbPC}}=K^{\mathrm{Xr}, \mathrm{XbPC}} \exp \frac{-\|i-l\|^{2}}{2\left(\sigma^{\mathrm{Xr}, \mathrm{XbPC}}\right)^{2}} \\
& \quad\left(\sigma^{\mathrm{Xr}, \mathrm{XbPC}}=1^{\circ}, K^{\mathrm{Xr}, \mathrm{XbPC}}=0.6\right) .
\end{aligned}
$$

The feedback connection from $\mathrm{Xb}_{\mathrm{PC}}$ to $\mathrm{Xr}$ has the same connection pattern with a different strength as follows:

$$
\begin{aligned}
w_{l m i}^{\mathrm{XbC}, \mathrm{Xr}}=K^{\mathrm{Xb}, \mathrm{Xr}} \exp \frac{-\|i-l\|^{2}}{2\left(\sigma^{\mathrm{XbP}, \mathrm{Xr}}\right)^{2}} \\
\quad\left(\sigma^{\mathrm{XbC}, \mathrm{Xr}}=1^{\circ}, K^{\mathrm{Xb}, \mathrm{Xr}}=3.0\right) .
\end{aligned}
$$

The connection weights between a cell $i$ in $\mathrm{Xe}_{\mathrm{PC}}$ and a cell $(l, m)$ in $\mathrm{Xb}_{\mathrm{PC}}$ are as follows:

$$
\begin{aligned}
& w_{i l m}^{\mathrm{X} e_{\mathrm{PC}, \mathrm{Xb}}}=K^{\mathrm{XePC}, \mathrm{XbpC}} \exp \frac{-\|i-m\|^{2}}{2\left(\sigma^{\mathrm{XePC}, \mathrm{XbPC}}\right)^{2}} \\
& \left(\sigma^{\mathrm{X} \mathrm{P}_{\mathrm{PC}, \mathrm{XbPC}}}=10^{\circ}, K^{\mathrm{XepC}_{\mathrm{P}, \mathrm{XbPC}}}=10.0\right)
\end{aligned}
$$

These connection matrices ensure that cell $(l, m)$ in the basis function map $\mathrm{Xb}_{\mathrm{PC}}$ is most strongly interconnected with cell $i=l$ in the retinotopic map Xr and $i=m$ in the internal eye position map $\mathrm{Xe}_{\mathrm{PC}}$.

The activity of the cells in the map is computed with the following:

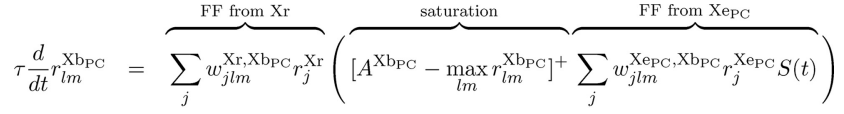

$$
\begin{aligned}
& +\underbrace{\sum_{j k} w_{j k l m}^{\mathrm{XbP}} r_{j k}^{\mathrm{Xb}}}_{\text {lat. excitation }}-\underbrace{\left(r_{l m}^{\mathrm{Xb}}+D^{\mathrm{Xb}}\right) w_{\mathrm{inh}}^{\mathrm{XbC}} \sum_{j k} r_{j k}^{\mathrm{XbC}}}_{\text {global inhibition }}-r_{l m}^{\mathrm{Xb}} \\
& \left(A^{\mathrm{X} b_{\mathrm{PC}}}=1.0, D^{\mathrm{X} b_{\mathrm{PC}}}=0.1, w_{\mathrm{inh}}^{\mathrm{Xb}_{\mathrm{PC}}}=0.4\right) .
\end{aligned}
$$

The excitatory weight between cells $(j, k)$ and $(l, m)$ is as follows:

$$
\begin{gathered}
w_{j k l m}^{\mathrm{XbpC}}=w_{\mathrm{exc}}^{\mathrm{Xr}} \exp \frac{-\left(\|j-k\|^{2}+\|l-m\|^{2}\right)}{\left(\sigma_{\mathrm{exc}}^{\mathrm{XbPC}}\right)^{2}} \\
\left(w_{\mathrm{exc}}^{\mathrm{XbpC}}=0.6, \sigma_{\mathrm{exc}}^{\mathrm{Xb}}=1^{\circ}\right) .
\end{gathered}
$$

We simulate perisaccadic suppression by a time-dependent factor $S(t)$ on the input from $\mathrm{Xe}_{\mathrm{PC}}$. The period of suppression is from $50 \mathrm{~ms}$ before to $32 \mathrm{~ms}$ after saccade offset. Given the latency of a stimulus, this leads to suppression of stimuli shown before saccade onset. During suppression, $S(t)=0.1$, and otherwise, $S(t)=1.0$.

Corollary discharge map $X e_{C D}$. We use a Gaussian input signal as follows:

$$
r_{i}^{\mathrm{XeCD}, \text { in }}(t)=S_{\mathrm{CD}}(t) c_{\mathrm{CD}} \exp \frac{-\left\|c_{i}^{\mathrm{Xe} C \mathrm{D}}-c^{\mathrm{CD}}\right\|^{2}}{2\left(\sigma^{\mathrm{CD}}\right)^{2}}
$$

to model the corollary discharge signal at the position $c^{\mathrm{CD}}$, the saccade target in retinotopic coordinates (or, equivalently, the planned saccade displacement), in the corollary discharge map $\mathrm{Xe}_{\mathrm{CD}}$, where $c_{i}^{\mathrm{Xe \textrm {CD }}}$ is the position of the receptive field center of cell $i$ in $\mathrm{Xe}_{\mathrm{CD}}$. The signal has the strength $c_{\mathrm{CD}}=0.25$, the width $\sigma^{\mathrm{CD}}=8^{\circ}$, and also a time course $S_{\mathrm{CD}}$ (see below). The ODE of the firing rate is as follows: 


$$
\tau \frac{d}{d t} r_{i}^{\mathrm{Xe} C \mathrm{D}}=r_{i}^{\mathrm{Xe},{ }_{\mathrm{CD}}^{\text {in }}}-r_{i}^{\mathrm{XeCD}}
$$

The time course $S_{\mathrm{CD}}$ of the transient CD signal is modeled by a Gaussian with different $\sigma$ for the rise $\left(S_{\mathrm{CD}}=S_{\mathrm{CD} \text {,on }}\right)$ and decay $\left(S_{\mathrm{CD}}=S_{\mathrm{CD} \text {,off }}\right)$ phases (Hamker et al., 2008) as follows:

$$
\begin{gathered}
S_{\mathrm{CD}, \text { on }}=\exp \frac{-\left\|t_{\mathrm{CD}}-t\right\|^{2}}{2\left(\alpha_{\mathrm{CD}}\right)^{2}} \quad\left(\alpha_{\mathrm{CD}}=50\right) \\
S_{\mathrm{CD}, \text { off }}=\exp \frac{-\left\|t_{\mathrm{CD}}-t\right\|^{2}}{2\left(\beta_{\mathrm{CD}}\right)^{2}} \quad\left(\beta_{\mathrm{CD}}=150\right) .
\end{gathered}
$$

$\left\|t_{\mathrm{CD}}-t\right\|$ is the time relative to the time of maximal CD activity. The maximum is at $t_{\mathrm{CD}}=10 \mathrm{~ms}$ after saccade onset. This value considers the time of the peak activity of movement-related cells in the FEF, which is typically at saccade onset (Sommer and Wurtz, 2004), plus the latency from the FEF to LIP, which is $\sim 2-12 \mathrm{~ms}$ (Ferraina et al., 2002). Since this arrival time in FEF is not known precisely, we use $10 \mathrm{~ms}$ as an estimate. However, in Results, we show that varying this latency by $\pm 10 \mathrm{~ms}$ does not change the model behavior.

Basis function map $\mathrm{Xe}_{F E F}$. The corollary discharge map $\mathrm{Xe}_{\mathrm{CD}}$ and the proprioceptive eye position map $\mathrm{Xe}_{\mathrm{PC}}$ are connected to the auxiliary basis function map $\mathrm{Xe}_{\mathrm{FEF}}$. The purpose of this intermediate map is to implement eye position gain fields on the retinotopic CD signal, as they have been found by Cassanello and Ferrera (2007). The corresponding matrix of connection weights between a cell $i$ in $\mathrm{Xe}_{\mathrm{CD}}$ and a cell $(l, m)$ in $\mathrm{Xe}_{\mathrm{FEF}}$ is as follows:

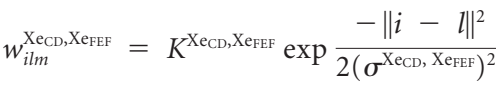

$$
\begin{aligned}
& \left(\sigma^{\mathrm{Xe}_{\mathrm{CD}}, \mathrm{Xe}_{\mathrm{EFF}}}=1^{\circ}, K^{\mathrm{X} \mathrm{X}_{\mathrm{CD}}, \mathrm{Xe}_{\mathrm{FEF}}}=0.5\right) .
\end{aligned}
$$

The connection weights between a cell $i$ in $\mathrm{Xe}_{\mathrm{PC}}$ and a cell $(l, m)$ in $\mathrm{Xe}_{\mathrm{FEF}}$ are as follows:

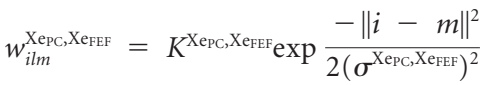

$$
\begin{aligned}
& \left(\sigma^{\mathrm{Xe}_{\mathrm{PC},} \mathrm{Xe}_{\mathrm{FeF}}}=2^{\circ}, K^{\mathrm{XePC}, \mathrm{Xe} \text { FFF }}=15\right) .
\end{aligned}
$$

These connection matrices ensure that cell $(l, m)$ in the basis function map $\mathrm{Xe}_{\mathrm{FEF}}$ is most strongly interconnected with cell $i=l$ in the corollary discharge map $\mathrm{Xe}_{\mathrm{CD}}$ and $i=m$ in the internal eye position map $\mathrm{Xe}_{\mathrm{PC}}$.

The activity $r_{l m}^{\mathrm{X} \text { e }}$ of of the cells in the map is computed by the following:

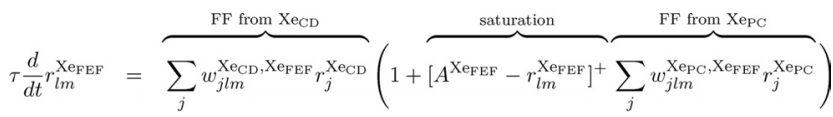

$$
\begin{aligned}
& -\underbrace{r_{l m}^{\mathrm{X}_{\mathrm{FEF}}} w_{\mathrm{inh}}^{\mathrm{X} \mathrm{e}_{\mathrm{FF}}} \sum_{j k} r_{j k}^{\mathrm{Xe}_{\mathrm{FEF}}}}_{\text {global inhibition }}-r_{l m}^{\mathrm{X}_{\mathrm{FEF}}} \\
& \left(A^{\mathrm{X} \mathrm{X}_{\mathrm{FEF}}}=1.0, w_{\mathrm{inh}}^{\mathrm{Xe}_{\mathrm{PEF}}}=0.2\right) .
\end{aligned}
$$

Basis function map $X b_{C D}$. The intermediate corollary discharge map $\mathrm{Xe}_{\mathrm{FEF}}$ and the retinotopic map $\mathrm{Xr}$ encoding the stimulus are connected to the second basis function map $\mathrm{Xb}_{\mathrm{CD}}$. The corresponding matrix of connection weights between a cell $i$ in $\mathrm{Xr}$ and a cell $(l, m)$ in $\mathrm{Xb}_{\mathrm{CD}}$ is as follows:

$$
\begin{aligned}
w_{i l m}^{\mathrm{Xr}, \mathrm{Xb}}=K_{C \mathrm{D}}^{\mathrm{Xr}, \mathrm{X} \mathrm{X}_{\mathrm{CD}}} \exp \frac{-\|i-l\|^{2}}{2\left(\sigma^{\mathrm{Xr}, \mathrm{Xb}}\right)^{2}} & \\
& \left(\sigma^{\mathrm{Xr}, \mathrm{Xb} b_{C D}}=1^{\circ}, K^{\mathrm{Xr}, \mathrm{Xb} b_{C D}}=2.0\right) .
\end{aligned}
$$

The connection weights between a cell $(i, k)$ in $\mathrm{Xe}_{\mathrm{FEF}}$ and a cell $(l, m)$ in $\mathrm{Xb}_{\mathrm{CD}}$ are as follows:

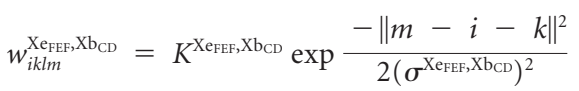

$$
\left(\sigma^{\mathrm{X}_{\mathrm{FFF}}, \mathrm{X} \mathrm{b}_{\mathrm{CD}}}=1^{\circ}, K^{\mathrm{X} \mathrm{X}_{\mathrm{FF}}, \mathrm{X} \mathrm{X}_{\mathrm{CD}}}=3.0\right) .
$$

In the head-centered model, the layer $\mathrm{Xb}_{\mathrm{CD}}$ receives feedback from $\mathrm{Xh}$. Then, the feedback connection from the head-centered cell $i$ to the basis function cell $(l, m)$ is as follows:

$$
\begin{aligned}
& w_{i l m}^{\mathrm{Xh}, \mathrm{Xb} \mathrm{bD}_{\mathrm{CD}}}=K^{\mathrm{Xh}, \mathrm{Xb} \mathrm{CD}} \exp \frac{-\|i-l-m\|^{2}}{2\left(\sigma^{\mathrm{Xh}, \mathrm{Xb} \mathrm{CD}}\right)^{2}} \\
& \left(\sigma^{\mathrm{Xh}, \mathrm{Xb} C \mathrm{CD}}=45^{\circ}, K^{\mathrm{Xh}_{\mathrm{Xb}} \mathrm{Xb}_{\mathrm{CD}}}=0.13\right) .
\end{aligned}
$$

In the non-head-centered model, $\mathrm{Xb}_{\mathrm{CD}}$ receives lateral input from $\mathrm{Xb}_{\mathrm{PC}}$. Then, the connection from the cell $(i, k)$ in $\mathrm{Xb}_{\mathrm{PC}}$ to the cell $(l$, $m)$ in $\mathrm{Xb}_{\mathrm{CD}}$ is as follows:

$$
\begin{aligned}
& w_{i k l m}^{\mathrm{Xb}_{\mathrm{pC}}, \mathrm{X} \mathrm{b}_{\mathrm{CD}}}=K^{\mathrm{Xb}_{\mathrm{PC}, \mathrm{Xb}}} \mathrm{b}_{\mathrm{CD}} \exp \frac{-\|i+k-l-m\|^{2}}{2\left(\sigma^{\mathrm{Xb}_{\mathrm{PC}, \mathrm{Xb} C \mathrm{D}}}\right)^{2}} \\
& \left(\sigma^{\mathrm{Xb}_{\mathrm{PC}, \mathrm{Xb}} \mathrm{B}_{\mathrm{CD}}}=47.4^{\circ}, K^{\mathrm{Xb}_{\mathrm{PC}, \mathrm{Xb}}}=0.16\right) .
\end{aligned}
$$

These connection matrices ensure that cell $(l, m)$ in the basis function map $\mathrm{Xb}_{\mathrm{CD}}$ is most strongly interconnected with cell $i=l$ in the retinotopic map Xr, $i+k=m$ in the intermediate corollary discharge map $\mathrm{Xe}_{\mathrm{FEF}}$, and cell $i=l+m$ in the head-centered map Xh.

The activity $r_{l m}{ }^{\mathrm{Xb}} \mathrm{CD}$ of the cells in the map is computed by the following:

$$
\begin{aligned}
& \tau \frac{d}{d t} r_{l m}^{\mathrm{Xb}}=\overbrace{\sum_{j} w_{j l m}^{\mathrm{Xr}, \mathrm{Xb}} b_{\mathrm{CD}} r_{j}^{\mathrm{Xr}}}^{\mathrm{FF} \text { from Xr }}(1+\overbrace{\left[A^{\mathrm{Xb}}-r_{l m}^{\mathrm{Xb}}\right]^{+}}^{\text {saturation }} \overbrace{\sum_{j k} w_{j k l m}^{\mathrm{Xe}_{\mathrm{FEF}}, \mathrm{Xb}_{\mathrm{CD}}} r_{j k}^{\mathrm{X}_{\mathrm{FEF}}}}^{\mathrm{FF} \text { from } \mathrm{X}_{\mathrm{FEF}}}) \\
& +\underbrace{\sum_{j} w_{j l m}^{\mathrm{Xe}}, \mathrm{Xb}_{\mathrm{CD}} r_{j}^{\mathrm{Xe}}}_{\mathrm{FF} \text { from Xe }} C \\
& -\underbrace{\left(r_{l m}^{\mathrm{Xb}}+D^{\mathrm{Xb}}\right) w_{\mathrm{inh}}^{\mathrm{Xb}_{\mathrm{CD}}} \sum_{j k} r_{j k}^{\mathrm{Xb}}}_{\text {global inhibition }}-r_{l m}^{\mathrm{Xb}_{\mathrm{CD}}} \\
& \left(A^{\mathrm{Xb}}=0.5, D^{\mathrm{Xb}_{\mathrm{CD}}}=0.1, w_{\mathrm{inh}}^{\mathrm{Xb}_{\mathrm{CD}}}=0.2\right) \text {. }
\end{aligned}
$$

Depending on the model variant, the connection term $C$ in the second line of Equation 25 is either $C=C_{\mathrm{Xh}}$ or $C=C_{\mathrm{XbP}}$.

$$
\begin{gathered}
C_{\mathrm{Xh}}=\underbrace{\sum_{j} w_{j l m}^{\mathrm{Xh}, \mathrm{Xb}} r_{j}^{\mathrm{Xh}}}_{\text {FB from Xh }} \\
C_{\mathrm{Xb}}=\underbrace{\sum_{i k} w_{i k l m}^{\mathrm{Xb}_{\mathrm{PC}}, \mathrm{Xb}_{\mathrm{CD}}} r_{i k}^{\mathrm{Xb}}}_{\text {lateral input from } \mathrm{Xb}_{\mathrm{PC}}}
\end{gathered}
$$

Different from classical basis function maps, the CD signal from the FEF $r_{l m}^{\mathrm{X} \mathrm{X}_{\mathrm{FF}}}$ modulates the gain in a rather attentive fashion from FEF to LIP [cf. Hamker (2005) for similar equations describing the effect of FEF on V4].

Head-centered map Xh (only in head-centered model). The headcentered map Xh is an optional map which only exists in the model version with an explicit head-centered representation. If enabled, it implements a head-centered response toward a visual stimulus. Thus, we have to ensure that a head-centered cell $i$ receives its strongest connection from the $\mathrm{Xb}_{\mathrm{PC}}$ cells $(l, m)$ for which $i=l+m$ holds as follows:

$w_{i l m}^{\mathrm{Xbp}, \mathrm{Xh}}=K^{\mathrm{XbPC}, \mathrm{Xh}} \exp \frac{-\|i-l-m\|^{2}}{2\left(\sigma^{\mathrm{XbPC}, \mathrm{Xh}}\right)^{2}}$

$$
\left(\sigma^{\mathrm{Xb}, \mathrm{Xh}}=15^{\circ}, K^{\mathrm{XbPC}, \mathrm{Xh}}=0.35\right) .
$$




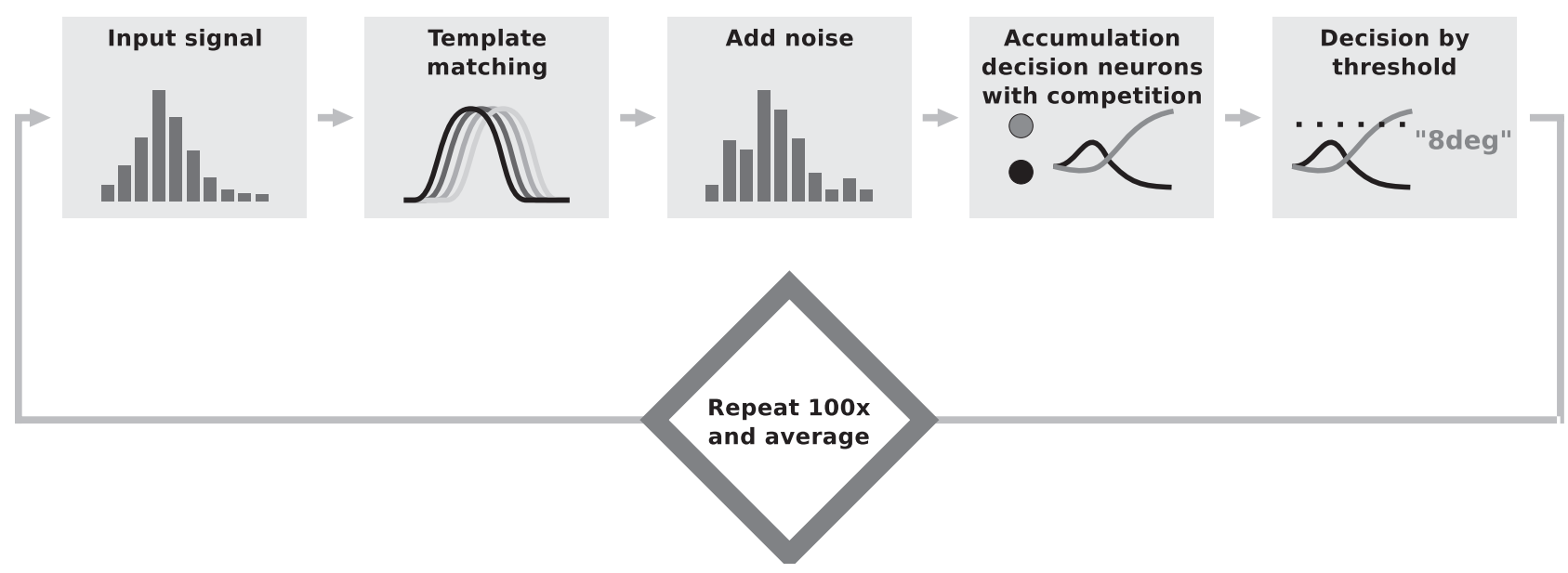

Figure 3. The decision process used in the model. The input signal that has to be decoded to yield a spatial position can either be the activity of the two simulated LIP layers $\mathrm{Xb}_{\mathrm{PC}}$ and $\mathrm{Xb}_{\mathrm{CD}}$ (in the non-head-centered model), or the activity of $\mathrm{Xh}$ (in the head-centered model). First, we apply a template matching for each time step. This has the advantage of increasing the spatial resolution by using appropriate precalculated templates. After that, noise is added so that not only the best matching template of each time step influences the final percept but the entire activity hill. These noisy template matches serve as input to accumulating decision neurons, which compete until a threshold is reached. We repeat the entire process 100 times to yield an average percept.

Similarly, it also receives input from the second $\mathrm{RBF}$ map $\mathrm{Xb}_{\mathrm{CD}}$ with a similar connection pattern to serve as a phasic "corrective" term around saccade onset as follows:

$$
\begin{aligned}
& w_{i l m}^{\mathrm{Xb}, \mathrm{Xh}}=K^{\mathrm{X} \mathrm{b}_{\mathrm{CD}}, \mathrm{Xh}} \exp \frac{-\|i-l-m\|^{2}}{2\left(\sigma^{\mathrm{Xb}, \mathrm{Xh}}\right)^{2}} \\
&\left(\sigma^{\mathrm{Xb}, \mathrm{Xh}}=15^{\circ}, K^{\mathrm{Xb}, \mathrm{Xh}}=0.2\right) .
\end{aligned}
$$

The head-centered stimulus response is dynamically encoded by a population of neurons, controlled by the ODE, as follows:

$$
\begin{gathered}
\tau \frac{d}{d t} r_{i}^{\mathrm{Xh}}=\underbrace{S_{i}^{\mathrm{Xh}} I_{i}^{\mathrm{Xh}}}_{\text {input }}+\underbrace{\sum_{j} w_{i j}^{\mathrm{Xh}} r_{j}^{\mathrm{Xh}}}_{\text {lat. excitation }}-\underbrace{\left(r_{i}^{\mathrm{Xh}}+D^{\mathrm{Xh}}\right) w_{\mathrm{inh}}^{\mathrm{Xh}} \sum_{j} r_{j}^{\mathrm{Xh}}}_{\text {global inhibition }}-r_{i}^{\mathrm{Xh}} \\
\left(D^{\mathrm{Xh}}=0.6, w_{\mathrm{exc}}^{\mathrm{Xh}}=0.2, \sigma_{\mathrm{exc}}^{\mathrm{Xh}}=1.0, w_{\mathrm{inh}}^{\mathrm{Xh}}=1.0\right) .
\end{gathered}
$$

Here,

$$
I_{i}^{\mathrm{Xh}}=\sum_{l m} w_{i l m}^{\mathrm{XbpCXh}} r_{l m}^{\mathrm{XbpC}}+\sum_{l m} w_{i l m}^{\mathrm{Xb} b \mathrm{Xh}} r_{l m}^{\mathrm{Xb} b_{C D}}
$$

is the input to the Xh neurons.

This input is subject to a synaptic depression $S_{i}^{\mathrm{Xh}}$ similar to the input of $\mathrm{Xr}$ neurons, although with a longer time constant $\tau_{S}^{\mathrm{Xh}}=10,000$ to allow for adaptation to stimuli that are present at the same head-centered position for several seconds $\left(d_{S}^{\mathrm{Xh}}=2.2\right)$, as typically observed in singlecell recordings.

$$
\begin{gathered}
\tau_{S}^{\mathrm{Xh}} \frac{d}{d t} s_{i}=I_{i}^{\mathrm{Xh}}-s_{i} \\
S_{i}^{\mathrm{Xh}}=1-d_{S}^{\mathrm{Xh}} s_{i} .
\end{gathered}
$$

The excitatory weight between cells $i$ and $j$ is as follows:

$$
w_{i j}^{\mathrm{Xh}}=w_{\mathrm{exc}}^{\mathrm{Xh}} \exp \frac{-\|i-j\|^{2}}{\left(\sigma_{\mathrm{exc}}^{\mathrm{Xh}}\right)^{2}} .
$$

Interpretation of neural activity using decision neurons. To compare the model performance with human performance, the neuronal activity of the model has to be mapped onto a perceptional decision. Rather than taking a particular snapshot in time we use a layer of "decision neurons" that accumulate evidence over time and compete for the final decision. In the non-head-centered model, the decision process receives input from both $\mathrm{Xb}_{\mathrm{CD}}$ and $\mathrm{Xb}_{\mathrm{PC}}$. In the head-centered model, the input comes from $\mathrm{Xh}$. The interpretation of the input to the decision process consists of several steps (Fig. 3 ) as follows.

(1) In the head-centered model, the input $I^{\mathrm{DP}}$ to the decision process consists of the firing rates of Xh (i.e., $I_{i}^{\mathrm{DP}}=r_{i}^{\mathrm{Xh}}$ ). In the non-headcentered model, a similar input is generated by the following:

$$
I_{i}^{\mathrm{DP}}=\sum_{l m} w_{i l m}^{\mathrm{XbPC}, \mathrm{DP}} r_{l m}^{\mathrm{Xb} P \mathrm{C}}+\sum_{l m} w_{i l m}^{\mathrm{Xb}, \mathrm{DP}} r_{l m}^{\mathrm{XbcD}}
$$

where the connection weights are also similar to those of Xh:

$$
\begin{aligned}
& w_{i l m}^{\mathrm{Xb}, \mathrm{DP}}=K^{\mathrm{XbPG}, \mathrm{DP}} \exp \frac{-\|i-l-m\|^{2}}{2\left(\sigma^{\mathrm{XbPG}, \mathrm{DP}}\right)^{2}} \\
& \left(\sigma^{\mathrm{XbPC}, \mathrm{DP}}=15.0, K^{\mathrm{Xb}, \mathrm{DP}}=0.035\right) \\
& w_{i l m}^{\mathrm{Xb}_{C \mathrm{D}} \mathrm{DP}}=K^{\mathrm{Xb} \mathrm{b}_{\mathrm{CD}}, \mathrm{DP}} \exp \frac{-\|i-l-m\|^{2}}{2\left(\sigma^{\mathrm{Xb} \mathrm{b}_{\mathrm{CD}}, \mathrm{DP}}\right)^{2}} \\
& \left(\sigma^{\mathrm{X} b_{C D}, \mathrm{DP}}=15.0, K^{\mathrm{X} b_{C D}, \mathrm{DP}}=0.02\right) .
\end{aligned}
$$

(2) The position information encoded in the input $I^{\mathrm{DP}}$ is decoded using template matching with precalculated templates of much higher spatial resolution than the number of entries in $I^{\text {DP }}$. Each entry codes $4^{\circ}$. Hence the templates represent stimulus position with a step size of $0.5^{\circ}$. Template matching is done using correlation. The match $m_{c}$ of the template $t_{i}^{c}$ representing a stimulus at position $c$ with neurons $i$ is as follows:

$$
m_{c}=\sum_{j} r_{j}^{\mathrm{Xh}} t_{j}^{c}
$$

The spatial resolution of the decision neurons equals that of the templates.

(3) We introduce noise by first transforming the rate coded input to a spiking neuron model using a Poisson spike train and then transforming it back to rate coded input by averaging. To be more specific, one time step of the input $m_{c}$ (the template match from the previous step) is equivalent to $n$ time steps of the spiking neuron $\tilde{m}_{c}(n=20$ is the bin size). Spiking is simulated in the simplest way: In each of the $n$ time steps, the neuron spikes if and only if $m_{c}>R s_{\max }$, where $R$ is a random number between 0 and 1 . The spiking activity of the neuron is $s_{\max }=1$ while the nonspiking activity is 0 . Thus the activity at the spiking time step $t$ is as follows: 


$$
\tilde{m}_{c}^{t}=\left\{\begin{array}{cl}
s_{\max }, & \text { if } m_{c}>R s_{\max } \\
0, & \text { else }
\end{array}\right.
$$

Then the activity of the spiking neuron is averaged to obtain a rate as follows:

$$
m_{c}=\frac{1}{n} \sum_{t=0}^{n-1} \tilde{m}_{c}^{t}
$$

(4) Accumulating decision neurons are implemented as in the study by Hamker (2007). The ODE of a decision neuron $d_{c}$ at position $c$ is as follows:

$$
\tau^{D N} \frac{t}{d t} d_{c}(t)=m_{c}\left(k+w_{\mathrm{exc}}^{\mathrm{DN}} d_{c}\right)+a w_{\mathrm{exc}}^{\mathrm{DN}} d_{c}-w_{\mathrm{inh}}^{\mathrm{DN}} d_{c} \sum_{j \neq c} d_{j},
$$

with time step $h^{\mathrm{DN}}=1$, time constant $\tau^{\mathrm{DN}}=50, k=3$, excitatory weight $w_{\text {exc }}^{\mathrm{DN}}=8.0$, and inhibitory weight $w_{\text {inh }}^{\mathrm{DN}}=0.1$. Each decision neuron $d_{c}$ is initialized with a baseline firing rate of 0.1 before the decision process begins. A decision is made once one of the neurons reaches the threshold $d_{\text {thresh }}=3000$. If none of the neuron reaches this threshold after $t_{\max }^{\mathrm{DN}}=$ $100 \mathrm{~ms}$, the neuron with the highest activity at that time wins. The parameters of the decision process are tuned so that normally a decision is reached slightly before $100 \mathrm{~ms}$ (Kiani et al., 2008).

(5) This whole process is repeated $c_{\text {trials }}^{\mathrm{DN}}=100$ times and then averaged over all trials.

Simulation of differential equations. The differential equations $\tau \frac{d}{d t} r(t)=I(t)-r(t)$ are simulated in an iterative fashion using the Euler method. Thus, the activity at the time $t+h$ is determined by $r(t+h)=r(t)+\frac{h}{\tau} I(t)$. We use a global network time constant $\tau=10$ ms and a global network time step of $h=1 \mathrm{~ms}$.

Simulation of saccadic eye movements. Saccadic eye movements are simulated by the same saccade model as in the study by Van Wetter and Van Opstal (2008). The eye position $E(t)$, evoked by a saccade target amplitude, $T$, is computed by the following:

$$
\begin{gathered}
E(t)=m_{0} \ln \frac{A \exp \left(v_{p k} t / m_{0}\right)}{1+A \exp \left(\left(v_{p k} t-T\right) / m_{0}\right.} \\
\text { with } A=\frac{1}{1-\exp \left(-T / m_{0}\right)},
\end{gathered}
$$

in which the parameters $m_{0}=7^{\circ}$ and $v_{p k}=525^{\circ} / \mathrm{s}$ determine the main sequence nonlinearity of the brainstem burst generator for eye velocity, $V(t)$, as follows:

$$
V(t)=v_{v p}\left(1-\exp \left(-R(T, t) / m_{0}\right)\right) .
$$

Here, $R(T, t)$ is the dynamic retinal error.

The simulated saccade is taken as ended once the eye velocity drops below $V_{\text {thresh }}=22 \%$. Then, the eye position is set to the saccade amplitude $T$.

Decoding eye position signals. The $\mathrm{RBF}$ layers $\mathrm{Xb}_{\mathrm{PC}}$ and $\mathrm{Xb}_{\mathrm{CD}}$ can be decoded to obtain an eye position signal for post hoc analysis of the model. For this, the sum of the layer (that is either $\mathrm{Xb}_{\mathrm{PC}}$, or $\mathrm{Xb}_{\mathrm{CD}}$, or the sum of both) is taken along the direction encoding the retinal position, so that only the eye position information remains. Thus, depending on the layer(s) to be decoded, define:

$$
\begin{aligned}
& r_{m}^{\mathrm{RBF}}=\sum_{l} r_{l m}^{\mathrm{PC}} \quad \text { or } \\
& r_{m}^{\mathrm{RBF}}=\sum_{l} r_{l m}^{\mathrm{CD}} \quad \text { or } \\
& r_{m}^{\mathrm{RBF}}=\sum_{l} r_{l m}^{\mathrm{PC}}+r_{l m}^{\mathrm{CD}} .
\end{aligned}
$$

Then this one-dimensional population $r_{m}^{\mathrm{RBF}}$ encoding spatial directions is extrapolated at both ends by repeating the values at these ends to get a population that encodes all directions from -180 to $180^{\circ}$. Let $\operatorname{deg}(m)$ be the preferred spatial direction of neuron $m$ in that population and let ind $(d)$ be its inverse (i.e., the index of the neuron with the preferred spatial direction $d$ ). Let $m_{0}$ be the neuron with the most leftward preferred spatial direction and $m_{1}$ the neuron with the most rightward preferred spatial location. Then define

$$
\begin{gathered}
r_{\text {ind }(d)}^{\mathrm{RBF}}=r_{m_{0}}^{\mathrm{RBF}} \quad \text { for all } d \text { with } \quad-180^{\circ}<d<\operatorname{deg}\left(m_{0}\right) \\
r_{\text {ind }(d)}^{\mathrm{RBF}}=r_{m_{1}}^{\mathrm{RBF}} \quad \text { for all } d \text { with } \operatorname{deg}\left(m_{1}\right)<d \leq 180^{\circ} .
\end{gathered}
$$

This extrapolation step has the purpose to cancel out equal activations across all neurons. Next, for each neuron $m$ a vector $v_{m}$ in twodimensional space is created whose direction is the spatial direction which the neuron encodes and whose length is the firing rate of the neuron.

$$
v_{m}=r_{m}^{\mathrm{RBF}}(\sin (\operatorname{deg}(m)), \cos (\operatorname{deg}(m))) .
$$

As a last step, the sum is taken over all these vectors to form a vector as follows:

$$
\bar{v}=\left(v_{x}, v_{y}\right)=\sum_{d=-180^{\circ}}^{180^{\circ}} v_{\operatorname{ind}(d)} .
$$

The direction

$$
\bar{d}=\tan ^{-1} \frac{v_{x}}{v_{y}}
$$

of the resulting vector $\bar{v}$ is the decoded eye position.

\section{Results}

\section{Cell properties}

We initially focus on the putative effects of corollary discharge and proprioceptive eye position on stimulus representation in LIP as simulated by $\mathrm{Xb}_{\mathrm{PC}}$ and $\mathrm{Xb}_{\mathrm{CD}}$ (Fig. $1 A$ ). Traces of the stimulus and eye position signals are shown in Figure $1 B . \mathrm{Xb}_{\mathrm{PC}}$ has classical gain field neurons whose firing rate is modulated by eye position from the proprioceptive signal (Fig. $1 C$, black and red lines). The cells in $\mathrm{Xb}_{\mathrm{CD}}$ do not depend on static eye position; they increase their response to stimuli due to the phasic CD. Even in the absence of visual stimulation in the classical receptive field, they respond to a combined signal of CD and lateral input from a joint representation of retinal stimulus position and proprioceptive eye position in $\mathrm{Xb}_{\mathrm{PC}}$ (Fig. $1 C$, green and blue lines). Both cell types $\left(\mathrm{Xb}_{\mathrm{PC}}\right.$ and $\left.\mathrm{Xb}_{\mathrm{CD}}\right)$ have been observed in the parietal cortex (Duhamel et al., 1992; Colby et al., 1996). Shortly before saccade onset when the $\mathrm{CD}$ raises (Fig. $1 B$ ), $\mathrm{Xb}_{\mathrm{CD}}$ updates its representation. $\mathrm{Xb}_{\mathrm{PC}}$ updates its representation much later. Changes in the proprioceptive signal start $\sim 50 \mathrm{~ms}$ after saccade offset (Fig. $1 B$ ). Around the same time, LIP cells that are modulated by the postsaccadic proprioceptive start responding (Fig. 1C, red line). However, since the presaccadic proprioceptive signal takes some time to decay (Fig. $1 B$, black line), on the population level a correct representation of eye position is restored even later, $\sim 200$ ms after saccade onset (see below, Decoding eye position). This is in line with recent electrophysiological findings in LIP (Xu et al., 2010).

To characterize in how far the two subpopulations of the simulated LIP cells are visually or motor driven, their responsiveness can also be plotted relative to stimulus onset on one axis and saccade onset on the other axis (Fig. 4). Such an analysis of cell 

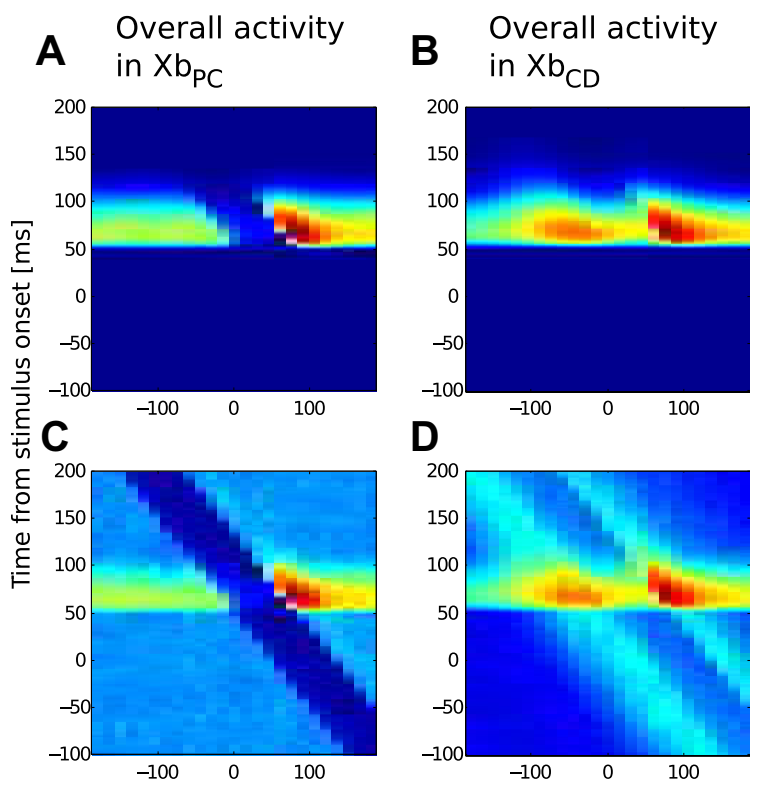

Figure 4. Activities of the two simulated LIP populations for $27^{\circ}$ saccades depending on stimulus onset time relative to saccade onset (on the $x$-axis) and time after stimulus onset ( $y$-axis), similar as in Bremmer et al. (2009). Each pixel shows the sum of activity across an entire neuron layer. Only one $15 \mathrm{~ms}$ flash is presented. $\boldsymbol{A}$, Activities of $\mathrm{Xb}_{\mathrm{pc}}$. The activation pattern has the form of a horizontal bar that spans a time from 50 to $100 \mathrm{~ms}$ after stimulus onset, indicating that this layer consists of visually driven neurons. The horizontal bar shows a diagonal interruption due to perisaccadic suppression in this layer. The suppression appears diagonally since presaccadic stimuli are only suppressed in the later part of their activity trace, whereas stimuli presented around saccade offset are only suppressed in the early part of their activity trace. $\boldsymbol{B}$, Activities of $\mathrm{Xb}_{\mathrm{CD}}$. It can be observed that this layer consists of visuomotor neurons. They are only active after a stimulus is presented; hence the overall pattern is also a horizontal bar. Different from $X b_{p c}$ neurons, activities here are modulated by corollary discharge; thus, they peak near saccade onset. Note that this layer also shows perisaccadic suppression, but only indirect via the lateral connections from $X \mathrm{Xb}_{\mathrm{PC}}$. $C$, Same as $A$, but with uniform noise of zero mean added to the retinal input signal. Here, the diagonal of the perisaccadic suppression is well observable. $\boldsymbol{D}$, Same as $\boldsymbol{B}$, but with noise. Here, the diagonal activation that shows that this layer is also motor driven. Also, the suppression diagonal that stems from lateral connections can be seen, although it is weaker than in $\mathrm{Xb}$ pc.

recordings has been performed recently by Bremmer et al. (2009). For better comparison, we show our model (top row) along with a model with additional noise on the visual input (bottom row). Figure 4, $A$ and $C$, shows that neuron activities in layer $\mathrm{Xb}_{\mathrm{PC}}$ are visually driven, only interrupted by a perisaccadic suppression phase (Bremmer et al., 2009). The cells in $\mathrm{Xb}_{\mathrm{CD}}$ (Fig. $4 B, D)$ do not depend on static eye position; they increase their response to stimuli due to the phasic CD. Thus, they show a saccade-related component. Bremmer et al. (2009) showed cells with even stronger saccade-related activity, but they preferably selected cells with a response to saccades. These cells are less affected by perisaccadic suppression. In our model, this is due to lateral input from $\mathrm{Xb}_{\mathrm{PC}}$ (i.e., from visual neurons). In conclusion, our model cells (in $\mathrm{Xb}_{\mathrm{PC}}$ and $\mathrm{Xb}_{\mathrm{CD}}$ ) show properties observed in cell recordings in LIP, which provides a solid basis for the following results.

\section{Saccade amplitude dependency}

In experiments that investigate the mislocalization of brief flashes in total darkness, a dependency of mislocalization on stimulus onset time relative to saccade onset has been observed. In these experiments, the subject has to perform a saccade from a fixation to a saccade target (Fig. $5 A$ ). Since such experiments typically require memory guided saccades, we also simulate eye move-

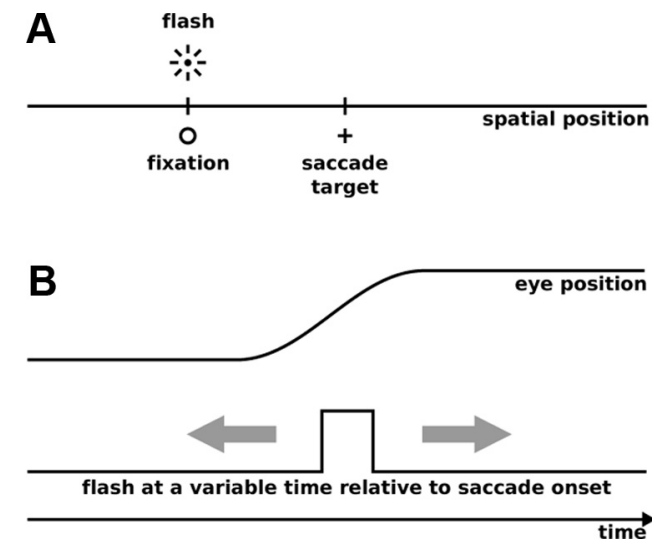

Figure 5. The experimental paradigm we simulate for perisaccadic shift in complete dark ness. $\boldsymbol{A}$, The spatial setup. Since misperceptions only occur parallel to the saccade direction, it is sufficient to simulate a one-dimensional world. In experiments, an elongated vertical bar is typically used. We simulate saccadic eye movements from fixation to saccade target. Stimulus flashes are presented at the fixation position, but neither our model nor experimental data show a strong dependence on flash position. In our simulations, as in the experiment of Van Wetter and Van Opstal (2008), we use four different saccade amplitudes $\left(9,14,27\right.$, and $\left.35^{\circ}\right) . \boldsymbol{B}$, The temporal setup. Flashes are presented at a variable time around the saccade between 180 $\mathrm{ms}$ before and $180 \mathrm{~ms}$ after saccade onset and with a varying duration $(5,15$, or $50 \mathrm{~ms})$.

ments without visual guidance. A brief stimulus flash (5, 15, or 50 $\mathrm{ms}$ in our simulations) is presented at the fixation position at a variable time between $180 \mathrm{~ms}$ before and $180 \mathrm{~ms}$ after saccade onset (Fig. 5B). The typical mislocalization curve peaks for stimuli flashed around the time of saccade onset (Fig. 6A). The amplitude of this peak mislocalization depends on the amplitude of the saccade. However, it does not scale linearly, as previously thought, but rather saturates around a level of $12^{\circ}$ even for long saccade amplitudes (Van Wetter and Van Opstal, 2008). Both versions, the non-head-centered model (Fig. $6 \mathrm{~B}$ ) and the headcentered model (Fig. 6C), replicate the saturation effect. Note, that, although we tuned parameters for the non-head-centered model, the head-centered model achieves a good data fit with the same parameters.

How does our model explain this saturation in the localization error (Fig. 6A)? A spatial percept is reached by a decision process that takes the activities of both LIP populations, $\mathrm{Xb}_{\mathrm{PC}}$ and $\mathrm{Xb}$ (or the head-centered layer Xh in the head-centered model) as its input. Thus, the decision process is driven by both, the proprioceptive and the CD signal. During fixation, it is only influenced by the retinal signal and the PC. Around saccade onset (i.e., when $\mathrm{Xb}_{\mathrm{CD}}$ increases its activity due to the $\mathrm{CD}$ signal), the activation pattern that serves as an input to the decoding process is more broadly tuned in position (the tuning, not the overall width of the population) for larger saccade amplitudes (Fig. 7). This is crucial for the ultimate percept. The proprioceptive signal, which updates late after the saccade, affects this activity with a stronger vote for the presaccadic eye position than the CD signal, which votes for the saccade target. In total, this leads to an average perception that is not at the arithmetic mean of both signals but closer to the presaccadic eye position. However, since for smaller saccades in which the activity hill in the decoded activity is more narrow, lateral competition lets both signals move toward each other yielding to a percept closer to the arithmetic mean (Fig. 8). Hence mislocalization does not scale linearly with saccade amplitude. Note that there is some variability between the models and the behavioral data for example in the earliest time of mislocalization or in the amount of negative mislocalization after saccade 
onset. Similar variability can also be observed between different conditions within the behavioral data. We will discuss possible sources of this variability below (see Parameter variations).

\section{Flash length dependency}

The perisaccadic shift also depends on flash duration (Jeffries et al., 2007; Van Wetter and Van Opstal, 2008). The main effect in the experimental data of Van Wetter and Van Opstal (2008) is that the localization error diminishes for longerlasting stimuli right at saccade onset (Fig. $6 D)$. Our model explains this by the typical accumulation time of $\sim 100 \mathrm{~ms}$ in the decision neurons. For longer flash durations, the neural representation of stimulus position in the later part of the neural response is more correct. This more reliable position information influences the ultimate decision in the accumulation process, a property of the non-head-centered model (Fig. 6E) as well as the head-centered model (Fig. $6 F$ ).

\section{Decoding eye position}

Previous models for perisaccadic mislocalization in total darkness often referred the extraretinal signal to an internal eye position estimate (psychophysical eye position). To relate our model to previous work, we decode an eye position signal directly from simulated LIP neurons in $\mathrm{Xb}_{\mathrm{PC}}$ and $\mathrm{Xb}_{\mathrm{CD}}$ (Fig. 9).

Compared with the true eye position from the saccade generator (Fig. 9, blue line), the decoded signal from proprioceptive $\mathrm{Xb}_{\mathrm{PC}}$ (red line) lags behind. A correct postsaccadic eye position representation is achieved as far as $150 \mathrm{~ms}$ after saccade offset, which is in line with recent findings (Xu et al., 2010). Thus, although the decoded signal from $\mathrm{Xb}_{\mathrm{CD}}$ (green line) gives an early vote for the new eye position, the signal from $\mathrm{Xb}_{\mathrm{PC}}$ votes for the old eye position until after saccade onset and even then it only slowly updates to the new eye position. Note that the signal decoded from $\mathrm{Xb}_{\mathrm{CD}}$ moves postsaccadically to a spatial position of almost twice the saccade amplitude. This effect is due to an updating of the CD signal stemming from modulatory influence from the proprioceptive signal in FEF. However, at this time, activities in $\mathrm{Xb}_{\mathrm{CD}}$ are already less than one-half of its maximum (Fig. 1C). Thus, this vote has little influence, which can also be seen in the combined decoding from both layers (Fig. 9, black line). However, we have no assumptions about the weight of each layer in such a combined decoding. It is possible that one of the neuron types far outnumbers the other, which would lead to a combined decoding that follows more closely either the signal decoded from proprioceptive or the signal decoded from CD cells. Given an equal weighting, the combined signal starts changing early and is slightly slower than the actual eye movement, thus having a time course similar to the classical extraretinal signal (Schlag and Schlag-Rey, 2002). However, after saccade offset, it overshoots the true eye position slightly. This effect is due to the influence of the proprioceptive signal on the simulated FEF. The signal also shows a transient retraction toward the initial fixation around 50-100 ms. This effect is due to the CD already reducing in strength while the proprioceptive still votes for an intermediate eye position. The overall time course of the combined signal is in line with recent physiological findings (Morris et al., 2010).

Note, however, that the decoded eye position signals cannot be directly compared with psychophysical signals of earlier models since the latter imply a far simpler interaction scheme. Hence the mislocalization patterns (Fig. $6 \mathrm{~B}$ ) cannot be deduced from decoded and true eye positions as in those models.

\section{Motor error}

The differentiation into two physiological signals, proprioception and corollary discharge, leads to novel predictions in the case of saccadic motor errors under the additional assumption that the CD signal encodes the initially planned saccade target, whereas the proprioceptive signal encodes after the saccade the actual landing point of the eye (Fig. 10A). Indeed, in several experimental setups, the saccade amplitude undershoots (i.e., is shorter than the vector to the saccade target) (Kapoula and Robinson, 1986). Thus, the mislocalization in the subpopulation of shorter saccades could be compared with those of normal saccade amplitude. Alternatively, saccade amplitude could be systematically altered by saccadic adaptation (McLaughlin, 1967). There is indeed some evidence that attention is also directed to the initially planned saccade endpoint after saccadic adaptation, which could suggest that the CD signal is directed to the planned location and not to the adapted location (Collins et al., 2010).

We simulated the effect of saccadic undershoots of 10 and $25 \%$ of the saccade amplitude on peak mislocalizations in the perisaccadic shift paradigm (Fig. $10 \mathrm{~B}$ ). We found that an undershoot of $10 \%$, which is a typical amount, leads to slightly increased peak mislocalizations while still showing the saturation behavior reported by Van Wetter and Van Opstal (2008). Interestingly, for a stronger undershoot of $25 \%$, which might be artificially created by saccadic adaptation, we can predict that peak mislocalizations only increase for large saccade amplitudes, thereby reducing the saturation effect. This is due to the CD 

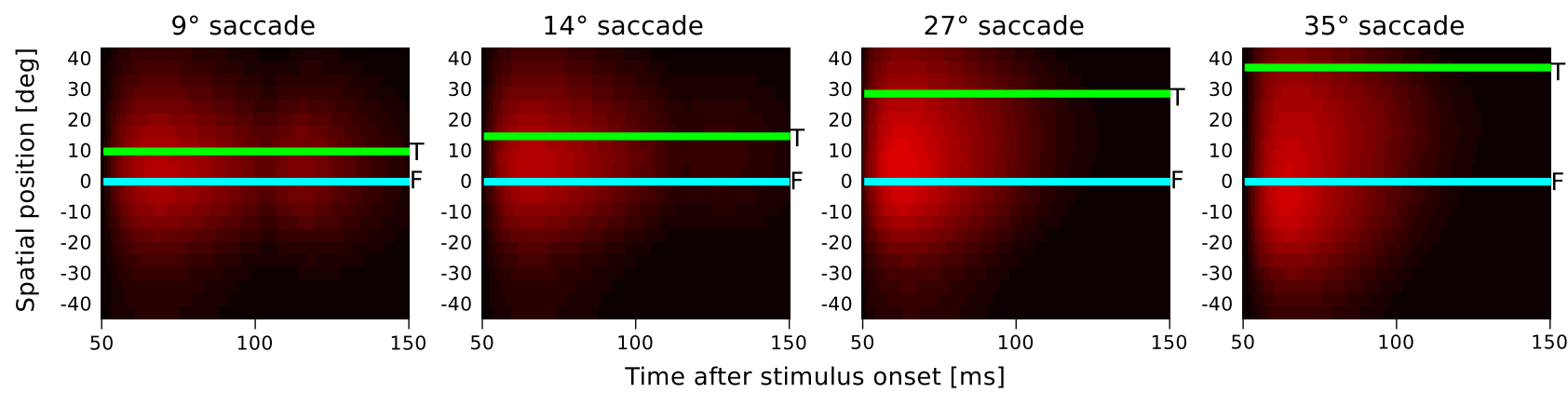

Figure 7. Activity traces used as the input signal in the decision process for different saccade amplitudes. Flash time has been chosen to produce the maximum amount of mislocalization. Stimuli were flashed at saccade onset for $15 \mathrm{~ms}$. The blue line (F) indicates the flash location. In the case of no mislocalization, the activity should be centered there. The green line indicates the saccade target that is the theoretical maximum amount of mislocalization.

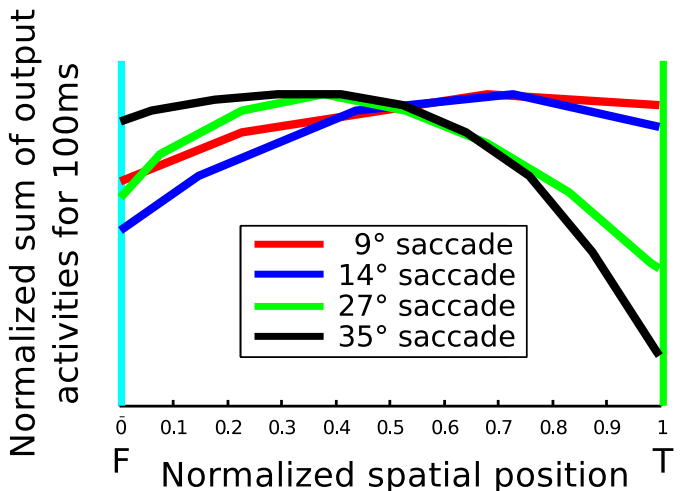

Figure 8. Average of the activity traces from Figure 7 over $100 \mathrm{~ms}$, which is the typical time until a perceptual decision is reached. Spatial position is normalized so that $\mathrm{F}$ and $\mathrm{T}$ are aligned for all saccade amplitudes. Activities are normalized to their maxima. The activity hill moves toward the fixation for higher saccade amplitudes, which explains the saturation effect.

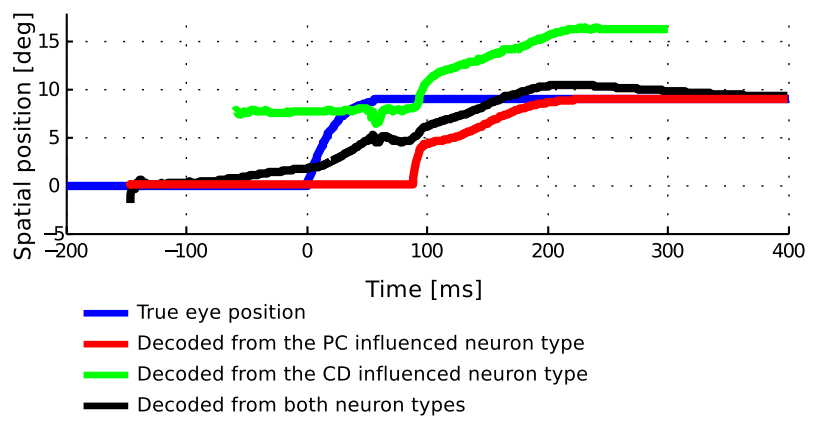

Figure 9. Comparing the true eye position from the saccade generator (blue) with eye position signals decoded from network layers for a $9^{\circ}$ saccade. The red and green lines are eye positions decoded from proprioceptive and $C D$ influenced neurons, respectively. The black lines are decoded from both neuron types. The signal decoded from both neuron types starts to move presaccadically and is slower than the actual eye position.

signal encoding the planned saccade amplitude, which implies that it does not depend on the motor error. At the time of peak mislocalization (i.e., around saccade onset), the CD signal induces a positive mislocalization that is the same, whether there is a motor error or not. However, around this time, the retinal signal starts to be affected by the beginning of the eye movement, which induces a movement of the perceived stimulus position toward a negative misperception (i.e., in the opposite direction of the eye movement). When there is a motor error, the saccade amplitude gets shorter and thus also the amount of movement toward negative misperception. In sum, there is more positive misperception in the case of motor error.
A
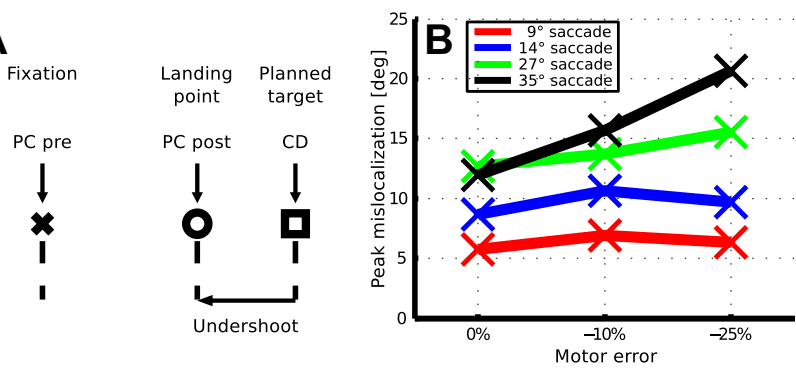

Figure 10. A, Simulated eye position signals in case of a saccadic motor error. We here assume that the $C D$ encodes the initially planned saccade target, whereas the proprioceptive signal encodes the actual landing point of the eye. If there is saccadic undershoot, the postsaccadic eye position signal and the CD will deviate. $\boldsymbol{B}$, The influence of saccadic undershoot on peak mislocalizations as predicted by our model. For large saccade amplitudes, the localization error increases with larger motor errors.

\section{Parameter variations}

The observed pattern of perisaccadic shift varies between studies. In particular, it has been found that misperceptions can also become more negative (i.e., opposite to saccade direction) after saccade onset (Honda, 1991). Similarly, the time where misperceptions start presaccadically varies between studies from -150 to $-50 \mathrm{~ms}$. We tested our model with respect to these variations by varying the time courses of the physiological input signals, namely the CD signal and proprioceptive signal (Fig. 11). For easier comparison, Figure $11 \mathrm{~A}$ replicates the outcomes of the non-head-centered model for saccade amplitude and flash length dependency from Figure 6, $B$ and $E$.

As a first variation, we were interested in the effect of the CD signal. We tested this by running a simulation in which we turned off the CD completely (Fig. 11C). As a result, there were almost no positive mislocalizations but strong negative mislocalizations immediately after saccade onset. There are some positive mislocalizations for the $9^{\circ}$ saccade (red line) but they only start as early as $30 \mathrm{~ms}$ before saccade onset. Since previous models claimed that perisaccadic shift can be explained without an anticipatory eye position signal (Pola, 2004; Teichert et al., 2010), we also tried to achieve the earliest possible mislocalizations without $\mathrm{CD}$ by shifting the updating of the proprioceptive signal to the earliest possible, although not plausible, time, which is immediately after saccade offset (data not shown). Indeed, this shifted the positive mislocalizations to start $\sim 50 \mathrm{~ms}$ before saccade onset, which is also the earliest time that mislocalizations started in the previous models by Pola (2004) and Teichert et al. (2010). An earlier misperception could only be achieved by a more pronounced visual latency or a longer (untypical) accumulation time in the decision 

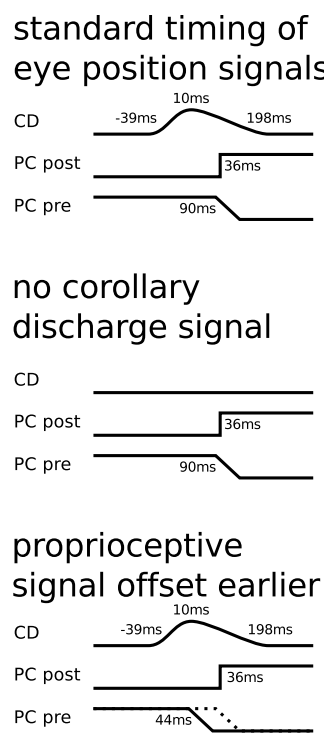

proprioceptive signal offset later

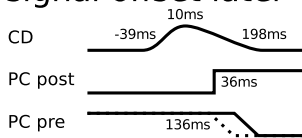

PC pre
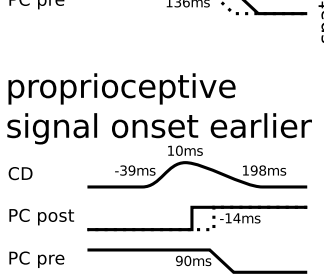

proprioceptive signal onset later

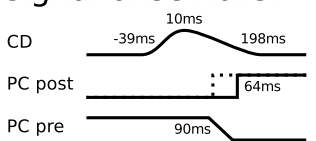

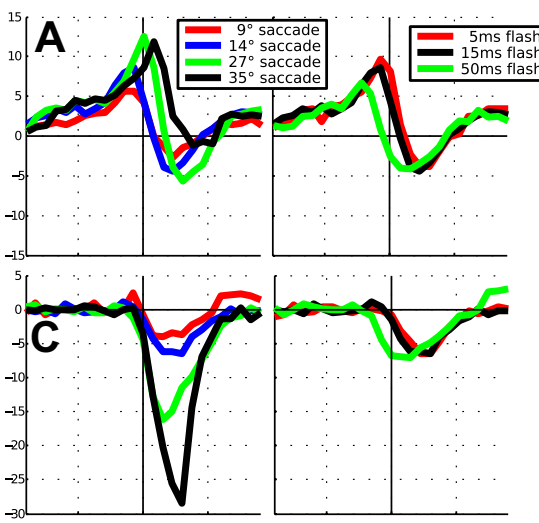
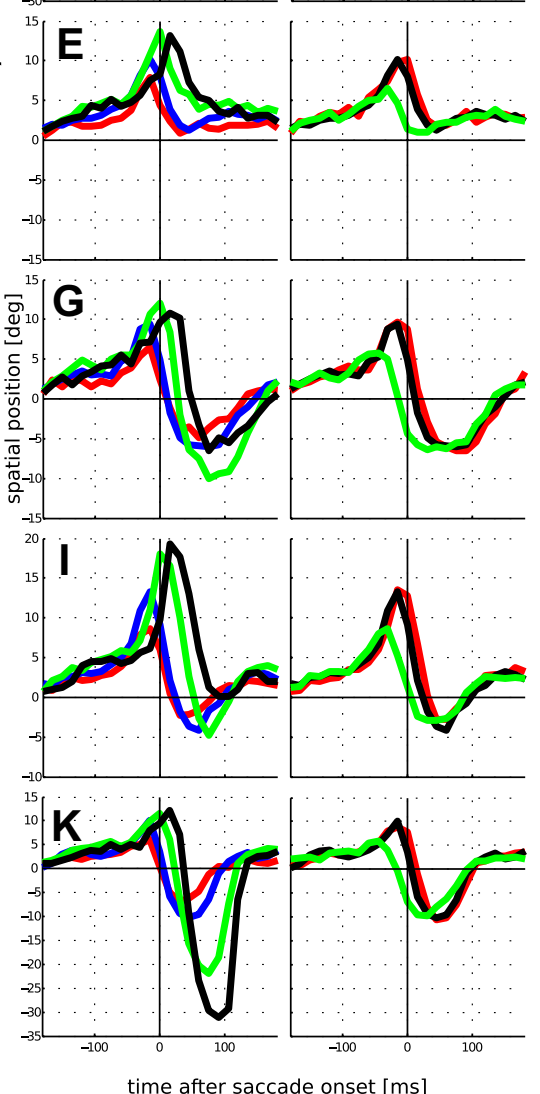

corollary discharge

signal earlier
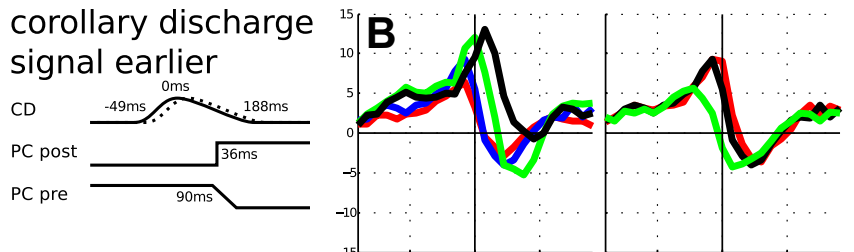

corollary discharge signal later
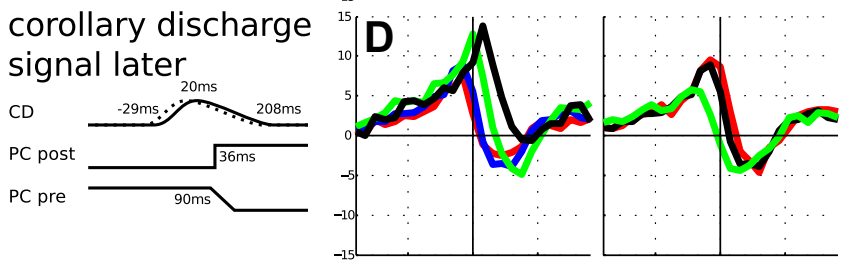

corollary discharge

signal onset earlier
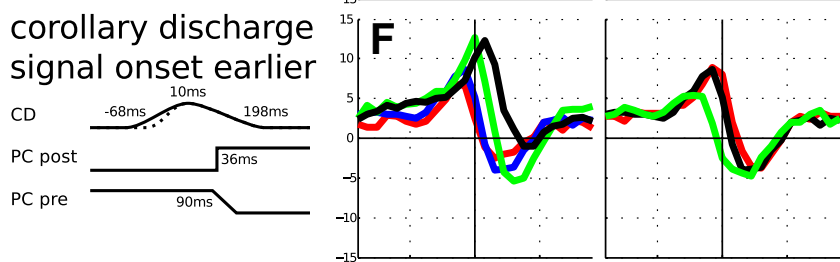

corollary discharge signal onset later
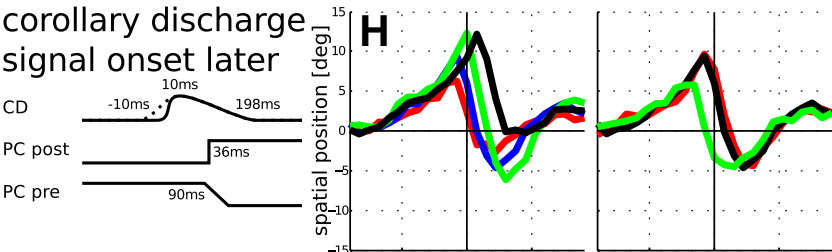

corollary discharge signal offset earlier
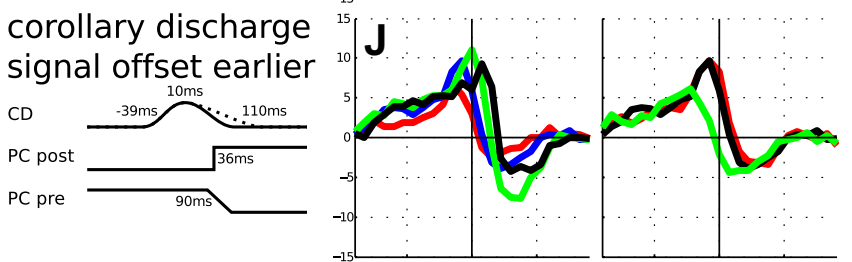

corollary discharge signal offset later
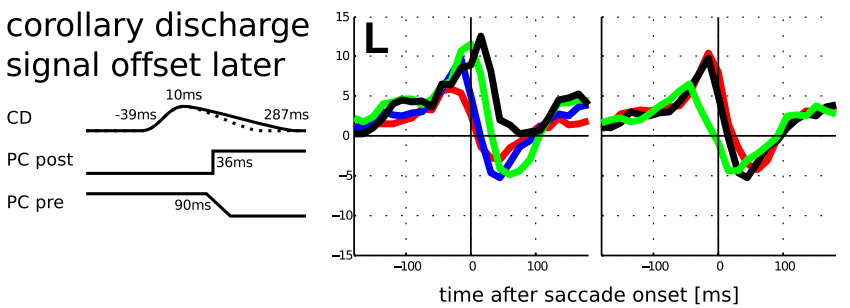

Figure 11. Parameter variations of the timing of the eye position signals for perisaccadic shift and flash length dependency using the non-head-centered model. The illustrations to the left of each panel show signal timings. Shown are times in which the signals reach one-half of their respective maxima and in which the CD signal peaks. Timings of the CD signal are relative to saccade onset; those of the proprioceptive signal are relative to saccade offset. $A$, The standard parameters of our model, same as in Figure $6, B$ and $E$. C, Outcomes with the same parameter set but without a $C D$ signal. It can be seen that the $C D$ signal is essential in explaining early mislocalizations. $B, D-L$, Variations of the timing parameters of the $C D$ and proprioceptive signals. The offset time of the presaccadic proprioceptive signal is responsible for the amount of negative mislocalization after saccade onset. The longer it is active, the stronger the negative mislocalizations are $(\boldsymbol{E}, \boldsymbol{G})$. The onset of the postsaccadic proprioceptive signal influences the peak mislocalization around saccade onset $(\boldsymbol{I}, \boldsymbol{K})$. The onset of the $(D$ signal controls the time the earliest mislocalizations appear $(\boldsymbol{F}, \boldsymbol{H})$. The offset of the $C D$ signal influences mislocalizations around saccade onset $(J, L)$. Note that, contrary to the proprioceptive signal timing $(E, K)$, the effect of $C D$ offset varies with saccade amplitude, with the most pronounced effect on the $35^{\circ}$ saccade. It also affects late mislocalizations beyond $100 \mathrm{~ms}$ after saccade onset. The dotted lines indicate the timing of parameters in $\boldsymbol{A}$.

neurons. Thus, according to our model, without the CD signal, it is not possible to explain the early mislocalizations that are observed in perisaccadic shift.

As far as the timing of the proprioceptive signal is concerned, it has recently been observed that proprioceptive eye position in LIP updates not immediately after saccade offset; rather complete updating can take as long as $150 \mathrm{~ms}$ (Xu et al., 2010). In our model parameters, we do not control the updating of the entire population but rather the offset and onset times of the signal at the presaccadic and postsaccadic eye positions. For the timing of the complete updating, see above (Decoding eye position). When we vary the time in which the presaccadic eye position signal turns off, we find that it primarily affects the amount of negative mislocalization after saccade offset (Fig. $11 E, G$ ). The later this offset time, the more negative the mislocalizations. As far as the timing of the onset of the new eye position signal at the postsaccadic eye position is concerned, we find primarily an influence on the peak mislocalizations around saccade onset. An earlier onset leads to stronger peak mislocalizations while a later onset leads to weaker peak mislocalizations as well as strong negative mislocalizations around saccade offset (Fig. $11 \mathrm{I}, \mathrm{K}$ ). A similar pattern can be observed, when the timing of the presaccadic and postsaccadic 


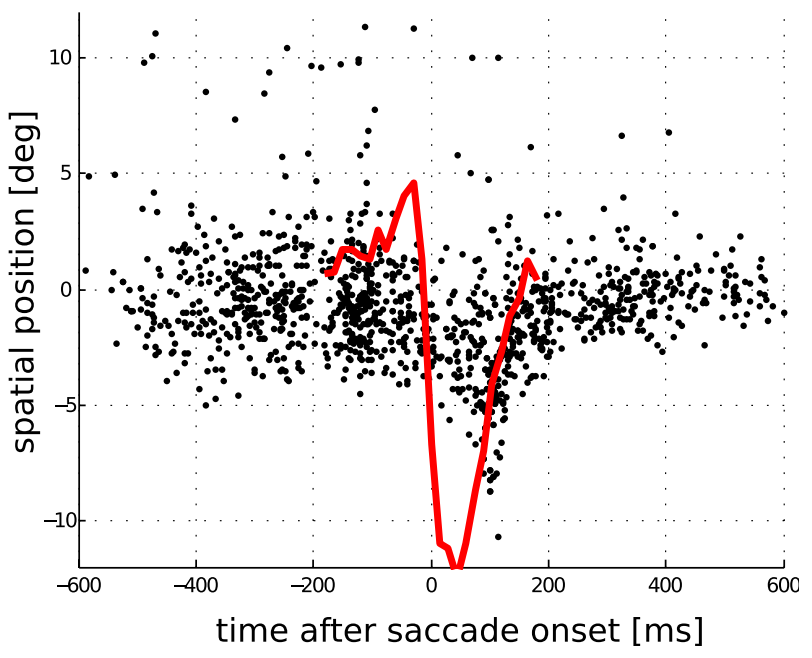

Figure 12. A later proprioception timing (offset and onset 50 ms later) combined with a weaker $C D$ signal (by a factor of 0.4 ) results in a stronger negative mislocalization as it is reported by Jeffries et al. (2007) for a $14^{\circ}$ saccade and $100 \mathrm{~ms}$ flash duration in a behavioral monkey study. The black dots are experimental data replotted from the study by Jeffries et al. (2007), their Figure $2 a$. The red line is model data.

signals is varied simultaneously (data not shown). In sum, this suggests that differences in the amount of negative mislocalization between studies stem from intersubject variability in the timing of proprioceptive eye position signals.

Figure $11, B, D, F, H, J$, and $L$, illustrates the effect of the timing of the CD signal. Varying the latency of the CD signal by $\pm 10 \mathrm{~ms}$ does not change the observed effects (Fig. $11 B, D$ ). The effect of the onset time of the CD signal is straightforward: the earlier the $\mathrm{CD}$ starts, the earlier is the mislocalization (Fig. $11 F, H$ ). The influence of the offset time of the CD signal is more subtle. In contrast to the timing of the proprioceptive signal, it has little influence on the perisaccadic mislocalization for small saccade amplitudes (the red and blue lines in Fig. 11, $J$ and $L$, are quite similar to those in Fig. $11 A$ ). However, long saccade amplitudes show an effect of CD offset timing on mislocalization (Fig. $11 \mathrm{~J}, L$, compare the peak mislocalizations of the black lines). Therefore, this parameter is partially responsible for the saturation behavior. This is in line with our earlier explanation of it (see above, Saccade amplitude dependency). Furthermore, the offset timing of the CD signal also affects late mislocalizations after $100 \mathrm{~ms}$ after saccade onset. Behavioral data sometimes show a second phase of positive mislocalization around that time (Fig. 6A). With a fast offset of the CD signal (Fig. $11 \mathrm{~J}$ ), these positive mislocalizations disappear, while with a longer decay they become stronger (Fig. $11 \mathrm{~L}$ ). In a monkey study, Jeffries et al. (2007) found almost no significant positive mislocalization presaccadically but a strong negative mislocalization after saccade onset. In their paper, they argued that this finding is inconsistent with a theory of a dampened eye position signal. Now, using our model, which invokes corollary discharge as well as proprioceptive eye position, we can show that these data (Fig. 12) can be well explained by a late updating of the proprioceptive signal (both offset of the presaccadic proprioceptive signal and onset of the postsaccadic proprioceptive signal $50 \mathrm{~ms}$ later) combined with a weaker CD signal (a factor of 0.4 ). The peak mislocalization in the model is a bit earlier than in the data. However, we did not intend to achieve an exact data fit by adjusting multiple parameters but rather provide an intuitive explanation for the peculiar observation of only negative mislocalization.

\section{Discussion}

Various phenomena of perisaccadic perception have been addressed by different models (Hamker et al., 2011). Our model proposed here suggests that the mislocalization of brief flashes in total darkness can be explained by proprioceptive and corollary discharge eye position information. This is different for flashes in light that are perceived closer to the saccade target rather than into the direction of the saccade vector (Ross et al., 1997). This pattern has been explained such that a corollary discharge, which serves for attentional gain control, locally increases the visual capacity around the saccade target (Hamker et al., 2008). Such attentional gain increase appears weak when the flashed stimulus is much brighter than the perceptual threshold, but might affect localization even in total darkness when the flashed stimulus is close to threshold (Georg et al., 2008). Furthermore, localization can be made relative to visual references, which explains that the strong shift in saccade direction is not observed in those studies. Thus, the localization of brief flashes in total darkness and under illumination seem to rely on a different use of extraretinal signals. Our model discussed here is only intended for conditions of total darkness and not when references come into play, as for example by using two successively flashed stimuli (Sogo and Osaka, 2002).

\section{Relationship to previous models}

Previous models of perisaccadic shift determined the craniocentric position of a flashed stimulus by subtracting an extraretinal eye position-related signal from a retinal position estimate. While early models (Dassonville et al., 1992) took a static account, deducing the extraretinal signal from behavioral data and interpreting it as a sluggish eye position estimate, Pola (2004) and Teichert et al. (2010) introduced a realistically modeled retinal signal and temporal integration and, as a consequence, concluded that an anticipatory extraretinal signal is not necessary to explain the data. However, these models struggle with early onsets of mislocalization as well as with newer findings that show a saturation of mislocalization for larger saccade amplitudes (Van Wetter and Van Opstal, 2008). Our model does not explicitly compute a retinal position estimate.

Another crucial difference to previous models is the process of perceptual decision making that is not only biologically more plausible than a simple time average within a temporal window but imposes additional constraints with respect to the properties of eye position signals.

\section{Eye position signals and their interaction}

Recently, proprioceptive and corollary discharge signals became the focus of several investigations (Sommer and Wurtz, 2004; Wang et al., 2007). Yet many open questions remain about their functions. The proprioceptive information is assumed to have at least a long-term calibration role, but its short-term relevance is unclear, as for example the double-step experiment appears to be doable using the corollary discharge alone (Guthrie et al., 1983). Corollary discharge is hypothesized to mediate perisaccadic suppression as well as predictive remapping of visual receptive fields to anticipate the effects of saccadic eye movements (Wurtz, 2008), but so far only little work demonstrated its relevance for performing particular tasks (e.g., double step) (Sommer and Wurtz, 2008). The model of Teichert et al. (2010) and similarly the one of Pola (2004) suggest that such anticipatory extraretinal signals are not required to explain the data of perisaccadic shift. However, we show that the more plausible assumption of perceptual decision making by a neural integration process instead of 
time averaging in previous models lessens the influence of the late trace of the stimulus persistence, since neural integration depends on the temporal order within the activity trace. By switching off the CD signal in our model, we show that with reafferent eye position information alone no mislocalizations earlier than $50 \mathrm{~ms}$ before saccade onset can be achieved, which is also the earliest possible mislocalization in the model of Teichert et al. (2010), even with slow temporal dynamics. Thus, we conclude that corollary discharge plays an essential role in explaining perisaccadic shift.

This leads to interesting predictions. If the CD signal provides an anticipatory signal for spatial updating, then a microstimulation of cells in the CD pathway during fixation should also lead to a mislocalization of flashed stimuli in total darkness. Moreover, when we artificially dissociate between the planned saccade target as encoded by the CD signal and the actual landing point of the eye encoded by the proprioceptive signal, the model predicts that the saturation effect decreases for larger saccade undershoots. This could be systematically tested within a saccadic adaptation paradigm.

\section{The role of gain fields and basis function networks}

Several parietal areas including LIP show gain fields, a dependency of the neural response of cells with retinocentric receptive fields on eye position. It has been suggested that such gain fields are involved in coordinate transformation to combine signals across different reference frames (Zipser and Andersen, 1988; Denéve et al., 2001; Salinas and Abbott, 2001; Pouget et al., 2002). Similar gain fields have also been motivated in other studies of spatial perception such as spatial updating in double saccade tasks (Keith et al., 2010). However, little is known about how gain fields are involved in online control and perisaccadic space perception. Recently, it has been observed that gain fields update eye position very late after saccade end (Xu et al., 2010), which suggests that they are not involved in online control. Our model is consistent with these data and proposes that both, gain fields and (attentional) modulations by a corollary discharge together, could represent the spatial percept as discussed before. However, such a unified spatial percept requires a combination of a signal that codes eye position in the orbit and another one for eye displacement, and thus, it is necessary that both signals are encoded within the same reference frame. One possible solution would be to implicitly combine eye displacement information with eye position information by gain fields, while still relying on retinocentric receptive fields. Some evidence suggests that the FEF already relies on such an implicit coding format (Cassanello and Ferrera, 2007). As shown in our model, this would be sufficient to merge both stimulus representations into a unifying percept.

It is controversially debated whether there are true headcentered stimulus representations within LIP (Mullette-Gillman et al., 2005) and how different reference frames are used for localization and reach planning (McGuire and Sabes, 2009). By implementing a model version with an explicit head-centered representation as the output layer, we can show that perisaccadic shift can be explained equally well in both cases without any further changes in the model.

In conclusion, it has been argued that corollary discharge helps in establishing a stable representation of the external world (Wurtz, 2008). While future computational work is required to provide testable predictions for the role of corollary discharge in visual stability, our present work suggests that corollary discharge indeed plays a substantial role in stimulus localization, at least when no visual references are used.

\section{References}

Andersen RA, Bracewell RM, Barash S, Gnadt JW, Fogassi L (1990) Eye position effects on visual, memory, and saccade-related activity in areas LIP and 7a of macaque. J Neurosci 10:1176-1196.

Bremmer F, Distler C, Hoffmann KP (1997) Eye position effects in monkey cortex. II. Pursuit- and fixation-related activity in posterior parietal areas LIP and 7A. J Neurophysiol 77:962-977.

Bremmer F, Kubischik M, Hoffmann KP, Krekelberg B (2009) Neural dynamics of saccadic suppression. J Neurosci 29:12374-12383.

Cassanello CR, Ferrera VP (2007) Computing vector differences using a gain field-like mechanism in monkey frontal eye field. J Physiol 582: 647-664.

Colby CL, Duhamel JR, Goldberg ME (1996) Visual, presaccadic, and cognitive activation of single neurons in monkey lateral intraparietal area. J Neurophysiol 76:2841-2852.

Collins T, Heed T, Röder B (2010) Visual target selection and motor planning define attentional enhancement at perceptual processing stages. Front Hum Neurosci 4:14.

Dassonville P, Schlag J, Schlag-Rey M (1992) Oculomotor localization relies on a damped representation of saccadic eye displacement in human and nonhuman primates. Vis Neurosci 9:261-269.

Denéve S, Latham PE, Pouget A (2001) Efficient computation and cue integration with noisy population codes. Nat Neurosci 4:826-831.

Duhamel JR, Colby CL, Goldberg ME (1992) The updating of the representation of visual space in parietal cortex by intended eye movements. Science 255:90-92.

Ferraina S, Paré M, Wurtz RH (2002) Comparison of cortico-cortical and cortico-collicular signals for the generation of saccadic eye movements. J Neurophysiol 87:845-858.

Galletti C, Battaglini PP, Fattori P (1993) Parietal neurons encoding spatial locations in craniotopic coordinates. Exp Brain Res 96:221-229.

Georg K, Hamker FH, Lappe M (2008) Influence of adaptation state and stimulus luminance on peri-saccadic localization. J Vis 8:15.1-11.

Guthrie BL, Porter JD, Sparks DL (1983) Corollary discharge provides accurate eye position information to the oculomotor system. Science 221:1193-1195.

Hamker FH (2005) The reentry hypothesis: the putative interaction of the frontal eye field, ventrolateral prefrontal cortex, and areas V4, IT for attention and eye movement. Cereb Cortex 15:431-447.

Hamker FH (2007) The mechanisms of feature inheritance as predicted by a systems-level model of visual attention and decision making. Adv Cogn Psychol 3:111-123.

Hamker FH, Zirnsak M, Calow D, Lappe M (2008) The peri-saccadic perception of objects and space. PLoS Comput Biol 4:e31.

Hamker FH, Zirnsak M, Ziesche A, Lappe M (2011) Computational models of spatial updating in peri-saccadic perception. Philos Trans R Soc Lond B Biol Sci 366:554-571.

Honda H (1989) Perceptual localization of visual stimuli flashed during saccades. Percept Psychophys 45:162-174.

Honda H (1991) The time courses of visual mislocalisation and of extraretinal eye position signals at the time of vertical saccades. Vis Res 31:1915-1921.

Jeffries SM, Kusunoki M, Bisley JW, Cohen IS, Goldberg ME (2007) Rhesus monkeys mislocalize saccade targets flashed for $100 \mathrm{~ms}$ around the time of a saccade. Vis Res 47:1924-1934.

Kapoula Z, Robinson DA (1986) Saccadic undershoot is not inevitable: saccades can be accurate. Vis Res 26:735-743.

Keith GP, Blohm G, Crawford JD (2010) Influence of saccade efference copy on the spatiotemporal properties of remapping: a neural network study. J Neurophysiol 103:117-139.

Kiani R, Hanks TD, Shadlen MN (2008) Bounded integration in parietal cortex underlies decisions even when viewing duration is dictated by the environment. J Neurosci 28:3017-3029.

Kusunoki M, Goldberg ME (2003) The time course of perisaccadic receptive field shifts in the lateral intraparietal area of the monkey. J Neurophysiol 89:1519-1527.

Matin L, Pearce E, Pola J (1970) Visual perception of direction when voluntary saccades occur: II. Relation of visual direction of a fixation target extinguished before saccade to a subsequent test flash presented before the saccade. Percept Psychophys 8:9-14.

McGuire LM, Sabes PN (2009) Sensory transformations and the use of multiple reference frames for reach planning. Nat Neurosci 12:1056-1061. 
McLaughlin S (1967) Parametric adjustment in saccadic eye movements. Percept Psychophys 2:359-362.

Morris AP, Kubischik M, Hoffmann KP, Krekelberg B, Bremmer F (2010) Dynamics of eye position signals in macaque dorsal areas explain perisaccadic mislocalisation. Paper presented at Vision Sciences Society Annual Meeting, Naples, FL, May.

Mullette-Gillman OA, Cohen YE, Groh JM (2005) Eye-centered, headcentered, and complex coding of visual and auditory targets in the intraparietal sulcus. J Neurophysiol 94:2331-2352.

Pola J (2004) Models of the mechanism underlying perceived location of a perisaccadic flash. Vis Res 44:2799-2813.

Pouget A, Denéve S, Duhamel JR (2002) A computational perspective on the neural basis of multisensory spatial representations. Nat Rev Neurosci 3:741-747.

Ross J, Morrone MC, Burr DC (1997) Compression of visual space before saccades. Nature 386:598-601.

Salinas E, Abbott LF (2001) Coordinate transformations in the visual system: how to generate gain fields and what to compute with them. Prog Brain Res 130:175-190.

Schlag J, Schlag-Rey M (2002) Through the eye, slowly: delays and localization errors in the visual system. Nat Rev Neurosci 3:191-215.

Sogo H, Osaka N (2002) Effects of inter-stimulus interval on perceived locations of successively flashed perisaccadic stimuli. Vis Res 42:899-908.

Sommer MA, WurtzRH (2004) What the brain stem tells the frontal cortex. I. Oculomotor signals sent from superior colliculus to frontal eye field via mediodorsal thalamus. J Neurophysiol 91:1381-1402.
Sommer MA, Wurtz RH (2008) Brain circuits for the internal monitoring of movements. Annu Rev Neurosci 31:317-338.

Stanford TR, Shankar S, Massoglia DP, Costello MG, Salinas E (2010) Perceptual decision making in less than 30 milliseconds. Nat Neurosci 13:379-385.

Tanaka M (2007) Spatiotemporal properties of eye position signals in the primate central thalamus. Cereb Cortex 17:1504-1515.

Teichert T, Klingenhoefer S, Wachtler T, Bremmer F (2010) Perisaccadic mislocalization as optimal percept. J Vis 10:19.

Usher M, McClelland JL (2001) The time course of perceptual choice: the leaky, competing accumulator model. Psychol Rev 108:550-592.

Van Wetter SMCI, Van Opstal AJ (2008) Experimental test of visuomotor updating models that explain perisaccadic mislocalisation. J Vis 8:8.1-22.

Wang X, Zhang M, Cohen IS, Goldberg ME (2007) The proprioceptive representation of eye position in monkey primary somatosensory cortex. Nat Neurosci 10:640-646.

Wurtz RH (2008) Neuronal mechanisms of visual stability. Vis Res 48: 2070-2089.

Xu Y, Karachi C, Goldberg ME (2010) The oculomotor system does not use LIP visual gain fields to calculate saccade target positions. Soc Neurosci Abstr 36:280.17.

Zipser D, Andersen RA (1988) A back-propagation programmed network that simulates response properties of a subset of posterior parietal neurons. Nature 331:679-684.

Zirnsak M, Beuth F, Hamker FH (2011) Split of spatial attention as predicted by a systems-level model of visual attention. Eur J Neurosci 33: 2035-2045. 